Portland State University

PDXScholar

7-8-1993

Detecting Chaotic Signals with Nonlinear Models

Qin Cai

Portland State University

Follow this and additional works at: https://pdxscholar.library.pdx.edu/open_access_etds

Part of the Signal Processing Commons

Let us know how access to this document benefits you.

Recommended Citation

Cai, Qin, "Detecting Chaotic Signals with Nonlinear Models" (1993). Dissertations and Theses. Paper 4564.

https://doi.org/10.15760/etd. 6448

This Thesis is brought to you for free and open access. It has been accepted for inclusion in Dissertations and Theses by an authorized administrator of PDXScholar. Please contact us if we can make this document more accessible: pdxscholar@pdx.edu. 
AN ABSTRACT OF THE THESIS OF Qin Cai for the Master of Science in Electrical Engineering presented July 8, 1993.

Title: Detecting Chaotic Signals with Nonlinear Models

APPROVED BY THE MEMBERS OF THE THESIS COMMITTEE:

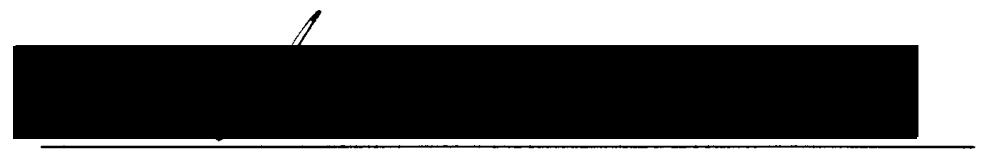

Andrew M. Fraser, Chair
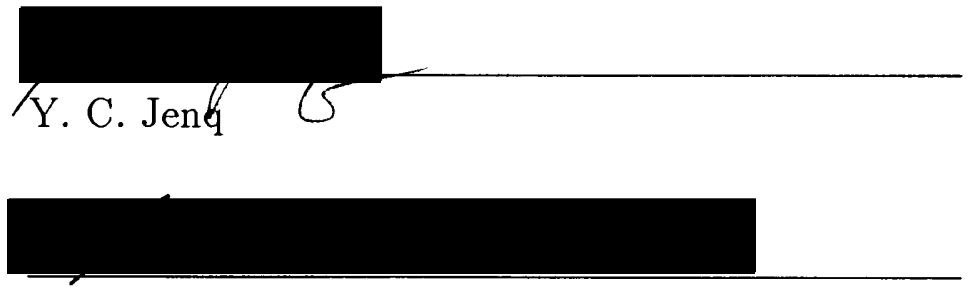

Beatrice T. Oshika

In this thesis we apply chaotic dynamic data analysis to the area of discrete time signal processing. A newly developed Hidden Filter Hidden Markov Model is introduced in detection of chaotic signals. Numerical experiments have verified that this novel nonlinear model outperforms linear AR model in detecting chaotic signals buried by noise having similar power spectra. A simple Histogram Model is proposed which can also be used to do detection on the data sets with chaotic behavior. Receiver Operating Characteristics for a variety of noise levels and model classes are reported. 


\title{
DETECTING CHAOTIC SIGNALS WITH NONLINEAR MODELS
}

\author{
by \\ QIN CAI
}

A thesis submitted in partial fulfillment of the requirements for the degree of

MASTER OF SCIENCE

in

ELECTRICAL ENGINEERING

\author{
Portland State University \\ 1993
}


TO THE OFFICE OF GRADUATE STUDIES:

The members of the committee approve the thesis of Qin Cai presented July 8, 1993.

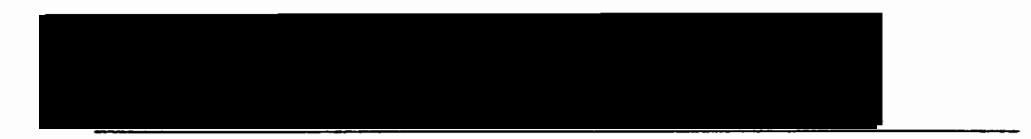

Andrew M. Fraser, Chair
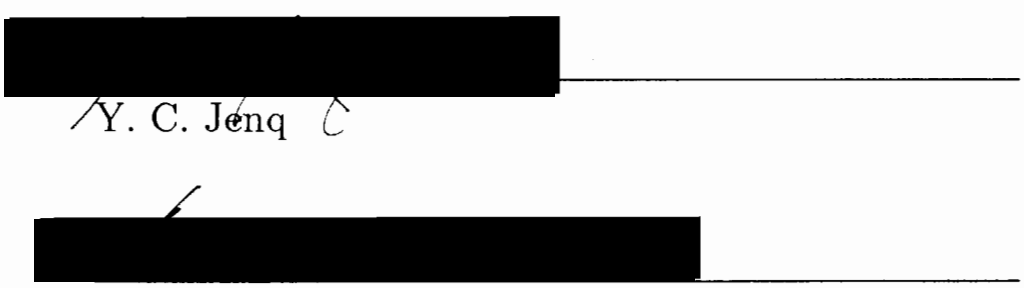

Beatrice T. Oshika

APPROVED:

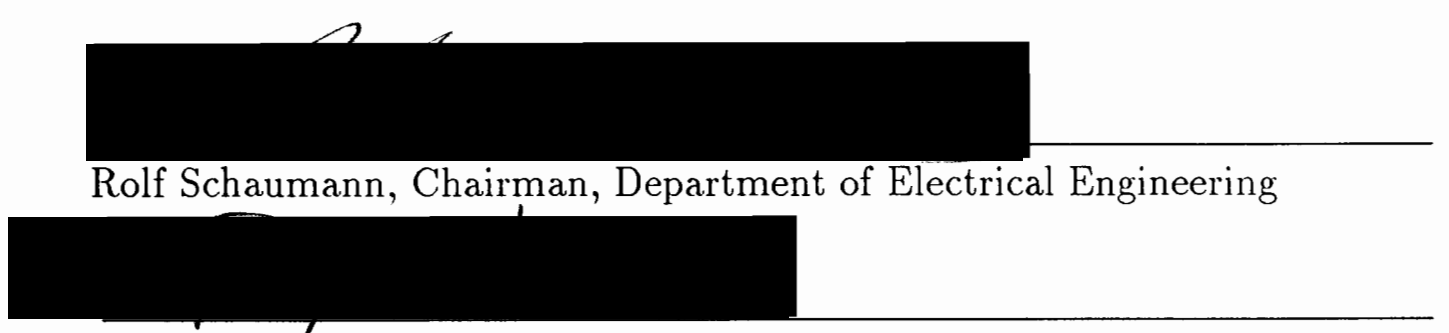

Roy W. Kokh, Vice Provost for Graduate Studies and Research 


\section{ACKNOWLEDGEMENT}

I wish to express my sincere gratitude to my advisor, Dr. Andrew M. Fraser, for his advice, encouragement and support in my graduate education.

I also acknowledge the National Science Fundation for its funding in nonlinear signal processing research.

My final thanks go to Dr. Oshika, Dr. Jenq, other faculty members and students at Portland State University for their valuble comments and contributions to the work presented here. 


\section{PREFACE}

I was fortunate to have the opportunity to work with Dr. Fraser when I arrived in the USA. First, I was asked to use existing software to detect signals which I knew nothing about. Through the work, I came to understand the concepts and methods of signal detection, learned about linear and nonlinear dynamic systems, and later realized the significance of the work we were doing. The whole learning process was a mix of fun and frustration. Thanks to the extreme patience of my adviser Dr. Fraser, and his guidance, I finally present the work here.

I used variations of the programs of the first Hidden Filter Hidden Markov Model (HFHMM1) written by Dr. Fraser and Alexis Dimitriadis for our detection experiments. In order to avoid the "overfit" problem, the number of model parameters should be as small as possible, which is the main reason Dr. Fraser suggested using a mapping $\mathbf{B}$ matrix in the AR functions to construct the HFHMM2. Instead of simply adopting Singular Value Decomposition (SVD) in linear fitting, the Conjugate Gradient Method is used as a searching algorithm. In the middle of studying the detection performance limit, I happened to find a simple Histogram Model (HM) which also can exploit chaotic properties of nonlinear systems. I developed 1D and high dimensional HMs which are described in chapter III in detail. Comparisons among different model classes including the AR model were made by plots of experimental Receiver Operating Characteristics. 
TABLE OF CONTENTS

PAGE

ACKNOWLEDGEMENT $\ldots \ldots \ldots \ldots \ldots \ldots$ iii

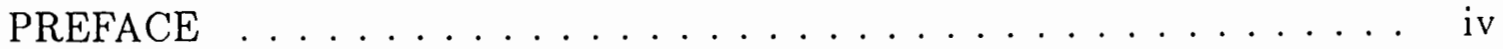

LIST OF TABLES ...................... vii

LIST OF FIGURES $\ldots \ldots \ldots \ldots \ldots \ldots \ldots$ viii

CHAPTER

I INTRODUCTION $\ldots \ldots \ldots \ldots \ldots \ldots \ldots \ldots$

I.1 Organization of the Thesis ............ 2

I.2 Notation . . . . . . . . . . . . . . . . 3

II REVIEW OF EXISTING DETECTION METHODS . . . . . . 7

II.1 The Detection Problem . . . . . . . . . . . . . 7

II.2 Detecting a Constant Signal in White Noise . . . . . . . 10

II.3 Using AR Spectral Estimation for Detection . . . . . . . . 13

II.4 Detecting Known Signals in White Noise . . . . . . . . . 18

III SIMPLE HISTOGRAM MODEL . . . . . . . . . . . . . . 26

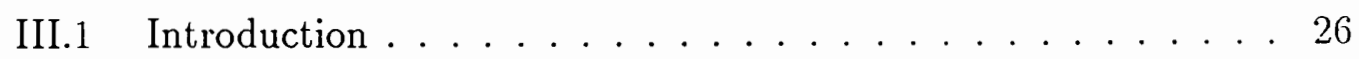

III.2 1D Histogram Model . . . . . . . . . . . . . . . 27

III.3 2D Histogram Model . . . . . . . . . . . . . . . . . 28

III.4 High Dimensional Histogram Model . . . . . . . . . . . . . 29

IV HIDDEN FILTER HIDDEN MARKOV MODEL $1 \ldots \ldots$

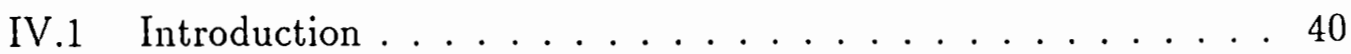


IV.2 Modeling . . . . . . . . . . . . . . 40

IV.2.1 Model Structure . . . . . . . . . . . . . . . 41

IV.2.2 The EM algorithm . . . . . . . . . . . . . 42

IV.2.3 HFHMMs . . . . . . . . . . . . . . . . . 43

IV.2.4 The Forward Backward Algorithm . . . . . . . . . . 46

IV.3 Detection of Chaotic Signals . . . . . . . . . . . 47

IV.3.1 Detection Methods . . . . . . . . . . . . . . 47

IV.3.2 Detection Experiments . . . . . . . . . . . . . 49

IV.3.3 Discussions ............... . 50

V HIDDEN FILTER HIDDEN MARKOV MODEL $2 \ldots \ldots$. . . . . . . 58

V.1 Introduction ................ 58

V.2 Modeling .................. 59

V.3 Detection of Chaotic Signals ............. 62

V.3.1 Detection Methods . . . . . . . . . . . . . . . 62

V.3.2 Experiment Results . . . . . . . . . . . . . . . 64

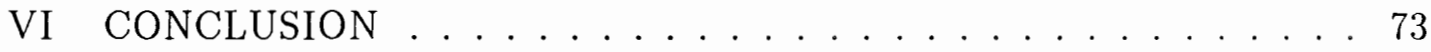

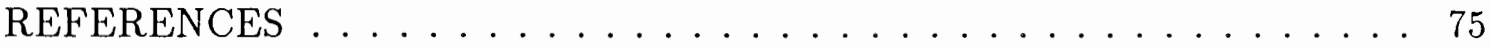




\section{LIST OF TABLES}

TABLE

PAGE

I Test Result Training on 4,000 Samples with 5 Hidden States by Starting from Delay Coordinates $\ldots \ldots \ldots \ldots 6$

II Test Result Training on 4,000 Samples with 5 Hidden States by Starting from Random Coordinates . . . . . . . . . . . . . . 66

III Comparison between SVD and CGM Training on 4,000 Samples with 5 Hidden States . . . . . . . . . . . . . . . . 67 


\section{LIST OF FIGURES}

FIGURE

PAGE

1 Components of a decision theory problem. . . . . . . . . 21

2 Error probabilities: (a) $P_{F}$ calculation, (b) $P_{D}$ calculation. . . . . . 22

3 (a) Signals: the Duffing System (thick), a 15th order AR noise (thin),

(b) PSD of the signal and noise (seen from the plot that they are very

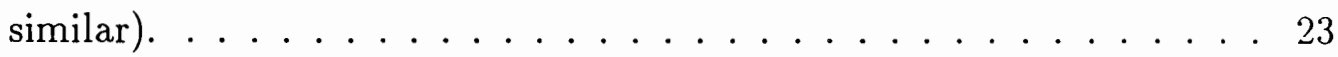

4 Experimental ROC for detecting the pure Duffing signal from the 15th order AR noise using 10th order AR models. . . . . . . . . . 24

5 Experimental ROCs for detecting the noisy Duffing signal in 15th order AR noise by using 30th order AR models. . . . . . . . . . 25

6 Plots of $y(t+1)$ vs. $y(t)$, (a) the Duffing signal, (b) the 15th order

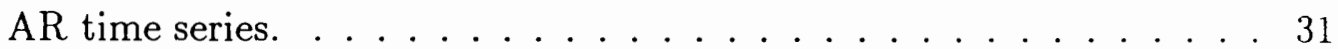

7 1D histogram with 47 bins and 4,000 length of training sequence, (a) the Duffing signal, (b) the 15 th order AR noise. . . . . . . . . . 32

8 1D histogram of mixed signal with 47 bins and 4,000 length of training sequence, (a) SNR $=0$, (b) SNR $=-9$, (c) SNR $=-17$, (d) SNR $=-30.33$

9 Experimental ROCs for detecting the noisy Duffing signal in 15th order AR noise using a 1D HM with 47 bins. . . . . . . . . . . 34

$102 \mathrm{D}$ histogram with $47^{2}$ bins and training sequence of length 20,000 . . 35

11 2D histogram of the noisy Duffing signal with $47^{2}$ bins and training sequence of length $20,000 \ldots \ldots \ldots \ldots$ 
12 Experimental ROCs for detecting the noisy Duffing signal in 15th order AR noise using 2D HM, where $47^{2}$ bins are used, the length of training and testing sequence is 20,000 and 4,000 respectively. . . . . 37

13 3D histogram with $47^{3}$ bins and training sequence of length 20,000 . . 38

14 Experimental ROCs for detecting the noisy Duffing signal in 15th order AR noise using 3D HM, where $47^{3}$ bins are used, the length of training and testing sequence is 20,000 and 4,000 respectively. . . . 39

15 Components of a HMM, $s_{i}$ and $s_{o}$ denote different hidden states, solid arrows denote the transition probabilities between these states, $y(t)$ denotes the scalar output which depends on the current states and previous samples. . . . . . . . . . . . . . . 52

16 Experimental ROCs for detecting the noisy Duffing signal in 15th order AR noise using different methods. . . . . . . . . . . 53

17 Different experimental ROCs using Method $\mathbf{I}$ at SNR of -24db, "length $T->M "$ denotes a model fit on length $T$ sequences and tested on length $M$ sequences, otherwise experiments are trained and tested on 4,000 samples. . . . . . . . . . . . . . . . . 54

$18 P(s, t)$ obtained from HFHMM1(3,5), (a) the Duffing signal, (b) the 15 th order AR noise. . . . . . . . . . . . . . 55

19 Output distribution $N\left(\bar{y}_{s}, \sigma_{s}^{2}\right)$ at state $s$, different line types denote different hidden states, (a) the Duffing signal, (b) the 15th order AR

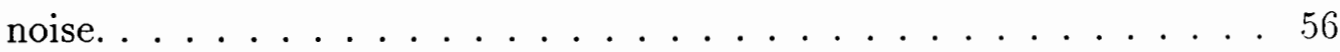

20 PSDs of AR models generated from $\mathbf{a}_{s},(a)$ the Duffing signal (state 1 and 4 are unplotted because of their unstability), (b) the 15th order

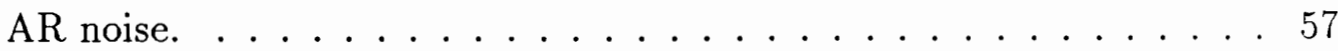


21 Experimental ROCs for detecting the noisy Duffing signal in 15 th order AR noise using Method I and model: (a) $\operatorname{HFHMM1}(6,10)$, (b) HFHMM2$(10 \rightarrow 3,10) \ldots \ldots . \ldots . \ldots . \ldots$

22 Experimental ROCs for detecting the noisy Duffing signal in 15 th order AR noise using Method I and HFHMM2(10 $\rightarrow 3,10)$. . . . . . . 69

23 Experimental ROCs for detecting the noisy Duffing signal in 15 th order AR noise using Method II and HFHMM2(10 $\rightarrow 3,10)$. . . . . . 70

24 Experimental ROCs for detecting the noisy Duffing signal in 15 th order AR noise using Method III and HFHMM2(10 $\rightarrow 3,10) . \quad$. . . . . 71

25 Experimental ROCs for detecting the noisy Duffing signal in 15 th order AR noise using Method IV and HFHMM2(10 $\rightarrow 3,10) . \quad$. . . . . 72 


\section{CHAPTER I}

\section{INTRODUCTION}

In this thesis the problem of detecting broad-band deterministic chaotic signals buried in noise having identical spectra has been studied. Well known spectral analysis techniques used in linear systems, such as the Autoregressive (AR) spectral estimator fail to distinguish this kind of noise from chaotic signals. A probabilistic Hidden Markov Model (HMM) approach [1] deals with such detection problems more effectively. The application of this method of detection builds a bridge between state space dynamics and discrete time signal processing.

Through the process of this study, chaotic signals from the Duffing Systems were chosen as the signal source. A suitable AR model was used to fit the power spectrum of this signal. The noise was then generated by driving this AR model, which resulted in the specific signal and noise having similar power spectrum. By using the programs written by Fraser and Dimitriadis [2] for the Hidden Filter Hidden Markov Model (HFHMM1), a Likelihood Ratio Test (LRT) was adopted as detector and obtained flawless experimental Receiver Operating Characteristics (ROCs) down to $-30 \mathrm{db}$. In order to avoid overfitting, a recently developed HFHMM2 was introduced which enhanced the performance of the previous HFHMM1 by adding a mapping matrix $\mathrm{B}$ in $\mathrm{AR}$ function. This helped to trace a longer history of the signal than the former algorithm. In chapter III, a simple Histogram Model (HM) which can achieve perfect experimental ROCs as low as -16 db was introduced, while 30 th order AR models reached only $2 \mathrm{db}$ and the AR models with order less than 
15 totally failed. In later chapters experimental ROCs for a variety of noise levels in different models were examined. Performance limits for special cases were studied and comparisons were made for different model classes.

\section{I.1 ORGANIZATION OF THE THESIS}

The thesis is organized as follows:

\section{- Chapter I: INTRODUCTION.}

This chapter includes an abstract and outline of the thesis, along with terminologies, operations, and basic symbols used frequently throughout the whole thesis.

- Chapter II: REVIEW OF EXISTING DETECTION METHODS.

This chapter defines what the detection problems are, summarizes several existing methods for detection problems, and examines the performance limit of detectors for different signals.

- Chapter III: SIMPLE HISTOGRAM MODEL

This chapter introduces a simple Histogram Model for detecting chaotic signals. The detection performance of experimental ROCs in 1,2 and 3 dimensional HMs are presented.

- Chapter IV: HFHMM1.

This chapter describes what a HFHMM1 is, and its use for detecting signals with similar PSD. Two kinds of methods are adopted in creating the model; one is training on the noisy data directly, the other is training on a noise free 
signal, then inflated for noise. Numerical experiments show that the detection performance obtained by using the latter method provides better results.

- Chapter V: HFHMM2.

This chapter explains how HFHMM2 is created based on the HFHMM1. Compared to the HFHMM1, it can trace the same length of previous samples with the use of fewer parameters. The two models trained on noisy data with the same number of parameters were tested, and the experimental ROCs are reported. The method of training a HFHMM2 on a noise free signal and then inflating for noise is also reported. In all cases HFHMM2 has better detection performance than HFHMM1, with the same number of parameters.

- Chapter VI: CONCLUSION

This chapter discusses the detection performance of different model classes and the problems left for further investigation.

\section{I.2 NOTATION}

Terminology:

ACF: Autocorrelation Function.

AR: Autoregressive.

CGM: The Conjugate Gradient Method.

EM: Estimate Maximize algorithm.

FBA: Forward Backward algorithm.

HMM: Hidden Markov Model.

HFHMM: Hidden Filter Hidden Markov Model. 
LRT: Likelihood Ratio Test.

MLE: Maximum Likelihood Estimate.

MSHMM: Mixed States Hidden Markov Model.

PDF: Probability Density Function.

PSD: Power Spectrum Density.

ROC: Receiver Operating Characteristics.

SNR: Signal to Noise Ratio.

SVD: Singular Value Decomposition.

VQ: Vector Quantization.

WSS: Wide Sense Stationary.

Operations:

\langle\rangle$_{p}$ : Expected value with respect to distribution $p$.

$(\cdot)^{T}$ : Transpose of matrix or vector $(\cdot)$.

$(\cdot)^{*}$ : Complex-conjugate of matrix or vector $(\cdot)$.

$(\cdot)^{-1}:$ Inverse of matrix $(\cdot)$

Basic Symbols:

$H_{1}$ : Hypothesis where signal is present.

$H_{0}$ : Hypothesis where only noise is present.

$\mathbf{R}^{n}: n$ dimensional real vector space.

$Z_{1}$ : Detection region under $H_{1}$. 
$Z_{0}$ : Detection region under $H_{0}$.

$P_{D}$ : Probability of Detection.

$P_{F}$ : Probability of False Alarm.

$P_{M}$ : Probability of Miss.

$N\left(m, \sigma^{2}\right)$ : Gaussian distribution with mean of $m$ and variance of $\sigma^{2}$.

$y(t)$ : The scalar observation at time $t$.

$y_{1}^{t}$ : The vector observation $[y(1), y(2), \ldots, y(t)]^{T}$.

$\mathbf{x}(t)$ : The history of $y(t)$, i.e. $y_{t-D}^{t-1}$.

$z(t)$ : The vector observation $y_{t-D}^{t}$.

$T$ : The number of time steps considered.

y: The vector observation $[y(1), y(2), \ldots, y(T)]^{T}$, i.e. $y_{1}^{T}$.

$\mathbf{X}$ : The matrix of histories, i.e. $[\mathrm{x}(1), \mathrm{x}(2), \ldots, \mathrm{x}(T)]^{T}$.

$\mathbf{v}(t)$ : Context vector at time $t$, i.e. $[v(t, 1), v(t, 2), \ldots, v(t, D)]^{T}$.

$S$ : Set of hidden states.

$s$ : An element of the set of hidden states $S$.

$N_{s}:$ Number of hidden states.

$q$ : A particular state sequence $s_{1}^{T}$.

Q: Set of all possible $q$. 
$s_{q}(t)$ : The discrete state assigned to time $t$ in the sequence $q$.

$\bar{y}_{s}$ : The constant part of HFHMM for the observation in state $s$.

a: The vector of AR coefficients.

$\mathbf{a}_{s}$ : The vector of AR coefficients associated with state $s$.

B: The mapping matrix $D \times H D$ in HFHMM2.

$\hat{y}_{s}(t)$ : The predicted value at time $t$ given state $s$ and history vector $\mathrm{x}(t)$, i.e. $\hat{y}_{s}(t)=$ $\bar{y}_{s}+\mathbf{a}_{s} \cdot \mathbf{x}(t)$ in HFHMM1, $\hat{y}_{s}(t)=\bar{y}_{s}+\mathbf{a}_{s} \cdot \mathbf{B x}(t)$ in HFHMM 2.

$\sigma_{s}:$ The standard deviation of $y$ for state $s$.

$\theta$ : Model parameters, such as $\sigma_{s}, \mathbf{a}_{s}$ and $\mathbf{B}$.

$P_{y(t) \mid s(t), \mathrm{x}(t), \theta}$ : The conditional PDF of $y$ given the current state and history vector at time $t$, i.e. $p_{y(t) \mid s(t), \mathbf{x}(t), \theta}: \mathbf{R} \times S \times \mathbf{R}^{D} \rightarrow \mathbf{R}$. The distribution is determined by the model parameters $\theta$.

$\operatorname{HFHMM1}\left(D, N_{s}\right): \operatorname{HFHMM} 1$ with order $D$ AR filters and $N_{s}$ discrete hidden states. HFHMM2 $\left(H D \rightarrow D, N_{s}\right)$ : HFHMM2 with higher order of $H D$ mapped to lower order of $D$ AR filters and $N_{s}$ discrete hidden states.

$r_{y y}(k):$ ACF of $y(t)$ with delay $k$.

$\mathbf{r}_{y y}:$ Vector of ACF of $y(t)$, i.e. $\left[r_{y y}(1), r_{y y}(2), \ldots r_{y y}(D)\right]^{T}$.

$r_{s}(k):$ ACF of $y(t)$ with delay $k$ associated with state $s$.

$\mathbf{r}_{s}$ : Vector of ACF of $y(t)$ in state $s$, i.e. $\left[r_{s}(1), r_{s}(2), \ldots r_{s}(D)\right]^{T}$. 


\section{CHAPTER II}

\section{REVIEW OF EXISTING DETECTION METHODS}

\section{II.1 THE DETECTION PROBLEM}

Detection theory has a long history. In detection problems we are required to choose among several possibilities for a given observation. In this thesis, only a binary hypothesis is considered. For example in well boring, a sharp drill is needed for efficient operation. However, once the drill is under the ground, it is hard to tell if it is blunt enough to warrant being changed. Since the installation costs money, time and labour, a correct decision needs to be made first. Audio data can be obtained while the drill is working and we need to discriminate the sound of a sharp drill head from that of a dull one. According to [3], the basic components of the detection problem are shown in Figure 1:

- Source:

It generates the output. For example we have two cases, $H_{1}$ where a signal is present and $H_{0}$ where there is only noise. Under the two hypotheses, suppose we have:

$$
\begin{aligned}
& H_{1}: \quad y(t)=y_{1}(t)+e(t) \\
& H_{0}: \quad y(t)=y_{0}(t)+e(t)
\end{aligned}
$$

here 
$y_{1}(t)$ : Signal at time $t$.

$y_{0}(t)$ : Noise at time $t$.

$e(t)$ : Noise caused by transition channel or other reason at time $t$.

- Probabilistic Transition Mechanism:

The transition mechanism can be viewed as a device which knows which hypothesis is true. In other words it is the model of conditional PDF $p\left(y_{1}^{T} \mid H\right)$ : $\mathbf{R}^{T} \rightarrow \mathbf{R}, H$ denotes the hypothesis, such as $H_{1}$ and $H_{0}$. For each given observation there exists a true hypothesis $H$, like the white Gaussian model we will discuss in next section. However in real practice, once you have received the signals, you do not know exactly what sources they come from. All you can do is to make several assumptions about the hypothesis, i.e., define what type of model you should choose in your detection problem, then use Maximum Likelihood Estimate (MLE) to evaluate the model parameters. So in numerical experiments we use $\hat{p}\left(y_{1}^{T} \mid H\right)$ instead of $p\left(y_{1}^{T} \mid H\right)$ in theory. This is the crucial part in a detection problem on which we should focus, i.e., building a good adaptive model to tell the difference between similar signals.

- Observation Space:

For each hypothesis, the probabilistic transition mechanism maps it to a point $y_{1}^{T}$ in observation space $Z$ which is the set of all these points. According to a decision rule discussed later in this section, the whole observation space $Z$ is divided into two parts, $Z_{0}$ and $Z_{1}$. Whenever an observation falls in $Z_{0}$ we guess that it represents case $H_{0}$, otherwise we say it is $H_{1}$.

- Threshold Device: 
Receiver Operating Characteristics (ROCs) are defined in terms of the following quantities:

$$
\begin{aligned}
P_{F} & =\int_{Z_{1}} p_{y \mid H_{0}}\left(Y \mid H_{0}\right) d Y \\
P_{D} & =\int_{Z_{1}} p_{y \mid H_{1}}\left(Y \mid H_{1}\right) d Y \\
P_{M} & =\int_{Z_{0}} p_{y \mid H_{1}}\left(Y \mid H_{1}\right) d Y=1-P_{D}
\end{aligned}
$$

where

$P_{F}$ : Probability of a false alarm, i.e. $Y$ is in $Z_{1}$ and $H_{0}$ is true.

$P_{D}$ : Probability of detection, i.e. $Y$ is in $Z_{1}$ and $H_{1}$ is true.

$P_{M}$ : Probability of a miss, i.e. $Y$ is in $Z_{0}$ and $H_{1}$ is true.

One can increase the probability of detection by lowering the alarm threshold which increases the size of $Z_{1}$ and decreases the size of $Z_{0}$. Lowering the alarm threshold also increases the probability of false alarm. A ROC is characterized by the plot of $P_{D}$ vs. $P_{F}$ that is generated by varying the alarm threshold. A good detector yields high $P_{D}$ with small $P_{F}$. Typical specification for $P_{D}$ and $P_{F}$ are 0.9 and 0.000001 respectively. See [4]. A diagonal straight line with 45 degree in plots of ROCs $\left(P_{D}\right.$ vs. $\left.P_{F}\right)$ means total failure of detection.

The Neyman-Pearson criterion, which maximizes $P_{D}$ with given $P_{F}$ by specifying a fixed threshold, leads us to use a Likelihood Ratio Test (LRT) to specify the regions $Z_{1}$ and $Z_{0}$

$$
\begin{aligned}
& H_{1} \\
& \Lambda(Y) \underset{<}{>} \eta \\
& H_{0}
\end{aligned}
$$


where $\Lambda(Y)$ is denoted as:

$$
\Lambda(Y) \stackrel{\text { def }}{=} \frac{P_{y \mid H_{1}}\left(Y \mid H_{1}\right)}{P_{y \mid H_{0}}\left(Y \mid H_{0}\right)}
$$

and $\eta$ is the threshold of the test. The notation in Equation 1 means that if $\Lambda(Y)>\eta$ then $Y \in Z_{1}$, and $H_{1}$ is reported. Otherwise $Y \in Z_{0}$, and $H_{0}$ is reported. As the natural logarithm is a monotonic function and both sides of Equation 1 are positive, an equivalent test is:

$$
\log \Lambda(Y) \stackrel{\begin{array}{l}
H_{1} \\
> \\
\\
H_{0}
\end{array}}{<} \log \eta
$$

This discussion assumes that we know what the sources are, or what the true hypotheses are. If we do not know $P_{y \mid H_{1}}$ and $P_{y \mid H_{0}}$, we use some estimation methods to evaluate model parameters $\theta_{1 / 0}$ from observations produced under $H_{1 / 0}$. Therefore we should rewrite:

$$
\Lambda(Y) \stackrel{\text { def }}{=} \frac{p_{y \mid \theta_{1}}\left(Y \mid \theta_{1}\right)}{p_{y \mid \theta_{0}}\left(Y \mid \theta_{0}\right)}
$$

To estimate the value of $\theta_{1 / 0}$ we usually use MLE trained on a sample sequence from $H_{1 / 0}$ by maximizing the conditional PDF $p_{y \mid \theta}(Y \mid \theta)$. Thus a general likelihood ratio test [5] is conducted and written as:

$$
\log \Lambda(Y)=\log \frac{p_{y \mid \theta_{1}}\left(Y \mid \theta_{1}\right)}{p_{y \mid \theta_{0}}\left(Y \mid \theta_{0}\right)} \underset{H_{0}}{<} \log \eta
$$

Equation 3 is the decision criterion we used in all detection experiments. 


\section{II.2 DETECTING A CONSTANT SIGNAL IN WHITE NOISE}

Detecting a constant signal corrupted by white Gaussian noise is the simplest case of a detection problem, and serves as a good example to illustrate how the LRT works. An example of this would be a digital communication system transmitting information by "ones" and "zeros". The two hypotheses are:

$$
\begin{aligned}
& H_{1}: \quad y(t)=m_{1}+e_{1}(t) \\
& H_{0}: y(t)=m_{0}+e_{0}(t)
\end{aligned}
$$

where

$m_{1}, m_{0}:$ Constants.

$e_{1}(t)$ : i.i.d. white Gaussian noise $N\left(0, \sigma_{1}^{2}\right)$.

$e_{0}(t)$ : i.i.d. white Gaussian noise $N\left(0, \sigma_{0}^{2}\right)$.

Because a Gaussian distribution is characterized only by mean and variance, we can easily find:

$$
\begin{aligned}
& p\left(Y \mid \theta_{1}\right)=\prod_{t=1}^{T} \frac{1}{\sqrt{2 \pi} \sigma_{1}} \exp -\frac{\left(y(t)-m_{1}\right)^{2}}{2 \sigma_{1}^{2}} \\
& p\left(Y \mid \theta_{0}\right)=\prod_{t=1}^{T} \frac{1}{\sqrt{2 \pi} \sigma_{0}} \exp -\frac{\left(y(t)-m_{0}\right)^{2}}{2 \sigma_{0}^{2}}
\end{aligned}
$$

For different $\sigma$ we have two cases:

1. When $\sigma_{1}=\sigma_{0}=\sigma$,

$$
\begin{aligned}
\log \Lambda(Y) & =\log \frac{\prod_{t=1}^{T} \frac{1}{\sqrt{2 \pi} \sigma} \exp -\frac{\left(y(t)-m_{1}\right)^{2}}{2 \sigma^{2}}}{\prod_{t=1}^{T} \frac{1}{\sqrt{2 \pi} \sigma} \exp -\frac{\left(y(t)-m_{0}\right)^{2}}{2 \sigma^{2}}} \\
& =\frac{m_{1}-m_{0}}{\sigma^{2}} \sum_{t=1}^{T} y(t)+\frac{m_{0}^{2}-m_{1}^{2}}{2 \sigma^{2}} T
\end{aligned}
$$


This example can be used to introduce the concept of sufficient statistic. When we construct a LRT $\Lambda(Y)$ which is a function of $Y$, in some cases it is possible for us to find a direct relationship with another function $L(Y)$ to make our calculation of $P_{D}$ and $P_{F}$ more obvious. Thus $\Lambda(Y)$ can be reduced to $\Lambda(L)$,

$$
\Lambda(L)=\frac{p_{l \mid H_{1}}\left(L \mid H_{1}\right)}{p_{l \mid H_{0}}\left(L \mid H_{0}\right)}
$$

where $L(Y)$ is called sufficient statistic. As for this example, we can define

$$
L(Y)=\frac{1}{\sqrt{T} \sigma} \sum_{t=1}^{T} y(t)
$$

Then Equation 2 in this example becomes

$$
L(Y) \underset{H_{0}}{\stackrel{H_{1}}{>}} \gamma \stackrel{\text { def }}{=} \frac{\sigma \log \eta}{\sqrt{T}\left(m_{1}-m_{0}\right)}+\frac{m_{1}+m_{0}}{2 \sigma} \sqrt{T}
$$

while $L(Y)$ is $N(0,1)$, and correspondingly

$$
\begin{gathered}
P_{D}=\int_{(\log \eta) / d+d / 2}^{\infty} \frac{1}{\sqrt{2 \pi}} \exp -\frac{(x-d)^{2}}{2} d x \\
P_{F}=\int_{(\log \eta) / d+d / 2}^{\infty} \frac{1}{\sqrt{2 \pi}} \exp -\frac{x^{2}}{2} d x
\end{gathered}
$$

where $d \stackrel{\text { def }}{=} \frac{\sqrt{T}\left(m_{1}-m_{0}\right)}{\sigma}$. The $P_{F}$ and $P_{D}$ in this case are shown in Figure 2 .

2. When $\sigma_{1} \neq \sigma_{0}$, the LRT is

$$
\sum_{t=1}^{T} \frac{(y(t)-m)^{2}}{k} \underset{H_{0}}{>} \gamma \stackrel{H_{1}}{>} \gamma \operatorname{def} \eta+\frac{\left(m_{1}-m_{0}\right)^{2}}{2} T+T \log \frac{\sigma_{1}}{\sigma_{0}}
$$

where

$$
m=\frac{m_{0} \sigma_{1}^{2}-m_{1} \sigma_{0}^{2}}{\sigma_{1}^{2}-\sigma_{0}^{2}} \quad k=\frac{2 \sigma_{1}^{2} \sigma_{0}^{2}}{\sigma_{1}^{2}-\sigma_{0}^{2}}
$$


We can define $L(Y)=\sum_{t=1}^{T}(y(t)-m)^{2}$, and the LRT becomes

$$
\begin{array}{cl}
L(Y) & \begin{array}{l}
H_{1} \\
>
\end{array} \\
& \gamma^{\prime} \stackrel{\text { def }}{=} k \gamma \quad\left(\sigma_{1}>\sigma_{0}\right) \\
& \\
& H_{0} \\
L(Y) & \stackrel{2}{>} \gamma^{\prime} \stackrel{\text { def }}{=} k \gamma \quad\left(\sigma_{1}<\sigma_{0}\right) \\
& < \\
&
\end{array}
$$

The performance calculation for $T>2$ is tedious. But if $T=2$ and $y(t)$ is i.i.d Gaussian, it is clear that $L(Y)>\gamma^{\prime}$ is the region outside a circle with center $(m, m)$ and radius $\sqrt{\gamma^{\prime}}$ on the condition of $k>0$, i.e. $\sigma_{1}>\sigma_{0}$. So

$$
\begin{aligned}
P_{F} & =P\left(L(Y) \geq \gamma^{\prime} \mid H_{0}\right) \\
& =\int_{0}^{2 \pi} d \phi \int_{\sqrt{\gamma^{\prime}}}^{\infty}(L+m) \frac{1}{2 \pi \sigma_{0}^{2}} \exp -\frac{\left(L+m-m_{0}\right)^{2}}{2 \sigma_{0}^{2}} d L \\
& =\exp \left(-\frac{\rho_{0}}{2 \sigma_{0}^{2}}\right)+\frac{\sqrt{2 \pi} m_{0}}{\sigma_{0}} \exp \left(-\frac{\rho_{0}^{2}}{4 \sigma_{0}^{2}}\right)
\end{aligned}
$$

and similarly

$$
\begin{aligned}
P_{D} & =P\left(l \geq \gamma^{\prime} \mid H_{1}\right) \\
& =\exp \left(-\frac{\rho_{1}}{2 \sigma_{1}^{2}}\right)+\frac{\sqrt{2 \pi} m_{1}}{\sigma_{1}} \exp \left(-\frac{\rho_{1}^{2}}{4 \sigma_{1}^{2}}\right)
\end{aligned}
$$

where

$$
\begin{aligned}
& \rho_{0}=\sqrt{\gamma^{\prime}}+m-m_{0} \\
& \rho_{1}=\sqrt{\gamma^{\prime}}+m-m_{1}
\end{aligned}
$$




\section{II.3 USING AR SPECTRAL ESTIMATION FOR DETECTION}

Before we do detection we should choose a model that returns a probability density given any sequence of observations. The White Gaussian Model used in the previous section is one of the choices, and is characterized by only two parameters, mean and variance. In the case that the signal and noise have the same mean and variance, but differ in other characteristics such as PSD, the white Gaussian Model will miss the detection, and other models are needed. The most popular of the time series modeling approachs to spectral estimation is the AR spectral estimator, which is superior to white Gaussian Model in this case. The PSD of an AR process depends on the AR parameters. See [6].

First we assume that observation $\mathbf{y}$ is Wide Sense Stationary (WSS), i.e., it has a constant mean $\langle y(t)\rangle$ and an Autocorrelation Function (ACF)

$$
r_{y y}(k)=\left\langle y^{*}(t) y(t+k)\right\rangle
$$

which depends only on the lag $k$ between the samples, not on their position $t$. An AR model can be described as:

$$
y(t)=\hat{y}(t)+e(t)
$$

where

$$
\hat{y}(t)=\mathbf{a} \cdot \mathbf{x}(t)
$$

is the estimated value of $y(t), x(t)=y_{t-D}^{t-1}$ is the history vector, $e(t)$ is the noise which is white Gaussian with $N\left(0, \sigma^{2}\right)$. AR coefficients a and observation noise variance $\sigma^{2}$ are evaluated to maximize

$$
p\left(y_{1}^{T} \mid \mathbf{a}\right)=\prod_{t=1}^{T} \frac{1}{\sqrt{2 \pi} \sigma} \exp -\frac{(y(t)-\mathbf{a} \cdot \mathbf{x}(t))^{2}}{2 \sigma^{2}}
$$


which is as same as minimizing $|\mathbf{y}-\mathbf{a} \cdot \mathbf{X}|^{2}$, where

$$
\sigma^{2}=\left\langle|y(t)-\hat{y}(t)|^{2}\right\rangle \approx \frac{1}{T}|\mathbf{y}-\mathbf{a} \cdot \mathbf{X}|^{2}
$$

The AR parameters can be obtained by solving the Yule-Walker Equations

$$
\mathbf{r}=\mathbf{R a}
$$

where

$$
\mathbf{R}=\left(\begin{array}{cccc}
r(0) & r(1) & \cdots & r(D-1) \\
r(1) & r(0) & \cdots & r(D-2) \\
\cdot & \cdot & \cdot & \cdot \\
\cdot & \cdot & \cdot & \cdot \\
\cdot & \cdot & \cdot & \cdot \\
r(D-1) & r(D-2) & \cdots & r(0)
\end{array}\right)
$$

Let $\mathbf{r}_{1}, \mathbf{r}_{0}$ be ACF of pure signal $s_{1}(t)$ and noise $s_{0}(t)$ respectively, and let $\mathbf{a}_{1}$ and $\mathrm{a}_{0}$ be the AR coefficients after fitting. So

$$
\begin{aligned}
& \mathbf{r}_{1}=\mathrm{R}_{1} \mathbf{a}_{1} \\
& \mathrm{r}_{0}=\mathrm{R}_{0} \mathbf{a}_{0}
\end{aligned}
$$

To simplify the calculation, we select the case $s_{1}(t)$ and $s_{0}(t)$ with the same observation noise power $\sigma^{2}$, i.e.

$$
r_{1}(0)-\sum_{i=1}^{D} a_{1}(i) r_{1}(i)=r_{0}(0)-\sum_{i=1}^{D} a_{0}(i) r_{0}(i)=\sigma^{2}
$$

Suppose $s_{1}$ and $s_{0}$ are uncorrelated. We assume

$$
\begin{aligned}
& H_{1}: y(t)=s^{\prime}(t)=\sqrt{\lambda} s_{1}(t)+\sqrt{1-\lambda} s_{0}(t) \\
& H_{0}: y(t)=s_{0}(t)
\end{aligned}
$$


with $0 \leq \lambda \leq 1$. The ACF of the two cases are:

$$
\begin{aligned}
& H_{1}: \mathbf{r}=\mathbf{r}^{\prime}=\lambda \mathbf{r}_{1}+(1-\lambda) \mathbf{r}_{0} \\
& H_{0}: \mathbf{r}=\mathbf{r}_{0}
\end{aligned}
$$

The Signal to Noise Ratio (SNR) is:

$$
S N R=10 \log _{10} \frac{\lambda}{1-\lambda}
$$

so

$$
\lambda=\frac{1}{1+10^{-S N R / 10}}
$$

For the mixed signal

$$
\begin{aligned}
\mathbf{r}^{\prime} & =\lambda \mathbf{r}_{1}+(1-\lambda) \mathbf{r}_{0} \\
& =\lambda \mathbf{R}_{1} \mathbf{a}_{1}+(1-\lambda) \mathbf{R}_{0} \mathbf{a}_{0} \\
& =\left[\lambda \mathbf{R}_{1}+(1-\lambda) \mathbf{R}_{0}\right] \mathbf{a}^{\prime} \\
& =\mathbf{R}^{\prime} \mathbf{a}^{\prime}
\end{aligned}
$$

and

$$
\sigma^{2}=[\lambda+(1-\lambda)] \sigma^{2}=\sigma^{2}
$$

Equation 10 leads to

$$
\mathbf{a}^{\prime}=\mathbf{R}^{\prime-1} \mathbf{r}^{\prime}
$$

Then under the two hypotheses:

$$
\begin{aligned}
& H_{1}: \quad y(t)=\mathbf{a}^{\prime} \cdot \mathbf{x}(t)+e(t) \\
& H_{0}: \quad y(t)=\mathbf{a}_{0} \cdot \mathbf{x}(t)+e(t)
\end{aligned}
$$


the LRT is

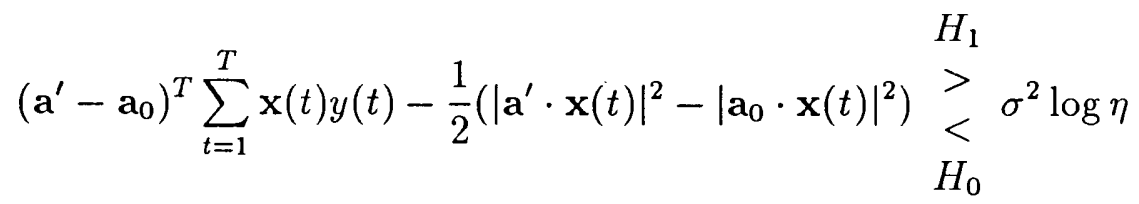

We estimate the ACF of $y(t)$ as follows:

$$
\begin{gathered}
r_{y y}(k)=\langle y(t) y(t+k)\rangle \\
\hat{r}_{y y}(k) \equiv \frac{1}{T} \sum_{t=1}^{T} y(t) y(t+k)
\end{gathered}
$$

Thus the LRT becomes

$$
\left(\mathbf{a}^{\prime}-\mathbf{a}_{0}\right) \cdot \hat{\mathbf{r}}_{y y}-\frac{1}{2} \sum_{i=1}^{D} \sum_{j=1}^{D}\left[a^{\prime}(i) a^{\prime}(j)-a_{0}(i) a_{0}(j)\right] \hat{r}_{y y}(i-j) \underset{H_{0}}{>} \frac{\sigma^{2}}{T} \log \eta
$$

Thus the difference of the expected values of the log likelihood is:

$$
\begin{aligned}
|\triangle \log L(Y)| & =\left|\log L\left(Y_{1}\right)-\log L\left(Y_{0}\right)\right| \\
& =\lambda\left|\triangle \mathbf{a} \cdot \triangle \mathbf{r}-\frac{1}{2} \sum_{i=1}^{D} \sum_{j=1}^{D} \triangle a_{i j} \triangle r(i-j)\right|
\end{aligned}
$$

where

$$
\begin{aligned}
& \triangle \mathbf{a} \stackrel{\text { def }}{=} \mathbf{a}^{\prime}-\mathbf{a}_{0} \\
& \Delta \mathbf{r} \stackrel{\text { def }}{=} \mathbf{r}_{1}-\mathbf{r}_{0} \\
& \Delta a_{i j} \stackrel{\text { def }}{=} a^{\prime}(i) a^{\prime}(j)-a_{0}(i) a_{0}(j)
\end{aligned}
$$

The difference between $\mathbf{a}^{\prime}$ and $\mathbf{a}_{0}$ decreases with $\lambda$. So the term $\mid \triangle \mathbf{a} \cdot \Delta \mathbf{r}-$ $\frac{1}{2} \sum_{i=1}^{D} \sum_{j=1}^{D} \triangle a_{i j} \Delta r(i-j) \mid$ is bounded. Therefore $|\triangle \log L(Y)|$ decreases with $\lambda$, which in turn is monotonic in the SNR. As SNR decreases, $|\triangle \log L(Y)|$ becomes narrower, and detection is more difficult. Equations 8 and 12 indicate that the 
performance of detection is entirely determined by the second order statistics of $\mathbf{y}$. Next we will verify that for our detection problem, an AR model fails if the only differences are in higher order statistics.

The following signal and noise sources are used in the numerical experiments throughout the rest of the thesis. We chose $u_{1}(t)$ from the Duffing System:

$$
\begin{aligned}
& \dot{u_{1}}=u_{2} \\
& \dot{u_{2}}=u_{1}-u_{1}^{3}-\delta u_{2}+\gamma \cos (t)
\end{aligned}
$$

as the source of the chaotic signal. We set $\gamma=0.275, \delta=0.25$ and set the sample frequency to 7 times the driving frequency. The noise was generated by driving a 15 th order AR model which has a similar spectrum to the signal. We used Ma.tlab for the linear methods in this work. Figure 3 shows that the PSDs of signal and noise are almost the same, but the sources are different.

A 10th order AR model totally misses the detection between pure signal and noise which is shown in Figure 4. For order greater than 15, AR models behave better because the ACF of the signal with lag larger than 15 is a small number other than zero while that of noise is zero. Figure 5 shows the experimental ROCs with SNR ranged from 2 to -10 by 30 th order AR models. compared to other model classes discussed in later chapters, the detection performance is still very poor.

\section{II.4 DETECTING KNOWN SIGNALS IN WHITE NOISE}

Consider the dull drill detection problem mentioned before in which the transmitter sends out one of the two signals $y_{0}(t)$ when the drill is dull and $y_{1}(t)$ when drill is still sharp and suppose the waveforms of the two cases are completely known. The signal at the receiver is corrupted by additive white Gaussian noise $e(t)$ which 
is $N\left(0, \sigma^{2}\right)$. The two hypotheses are:

$$
\begin{aligned}
& H_{1}: \quad y(t)=y_{1}(t)+e(t) \\
& H_{0}: y(t)=y_{0}(t)+e(t)
\end{aligned}
$$

It is easy to get

$$
\begin{aligned}
& p\left(Y \mid \theta_{1}\right)=\prod_{t=1}^{T} \frac{1}{\sqrt{2 \pi} \sigma} \exp -\frac{\left(y(t)-y_{1}(t)\right)^{2}}{2 \sigma^{2}} \\
& p\left(Y \mid \theta_{0}\right)=\prod_{t=1}^{T} \frac{1}{\sqrt{2 \pi} \sigma} \exp -\frac{\left(y(t)-y_{0}(t)\right)^{2}}{2 \sigma^{2}}
\end{aligned}
$$

and the LRT becomes

$$
\mathrm{y}^{T}\left(\mathrm{y}_{1}-\mathrm{y}_{0}\right) \underset{\substack{H_{1} \\ H_{0}}}{>} \sigma^{2} \log \eta+\frac{1}{2}\left(\mathrm{y}_{1}^{T} \mathrm{y}_{1}-\mathrm{y}_{0}^{T} \mathrm{y}_{0}\right)
$$

See $[5]$.

In later chapters, we use output from the Duffing System as the signal and output from a 15th order AR model as noise. The AR model is selected so that it has a similar spectrum to the signal. As described before, if two sources have similar PSD and the same power, the AR model is useless in this detection problem. The models we discuss later were designed to "see" these differences which AR models miss. Though in our detection problem, the waveforms of the signal and noise are unknown. Theoretically if we assume that we know them we can derive the detection performance. The two hypotheses are shown as follows:

$$
\begin{aligned}
& H_{1}: y(t)=\sqrt{\lambda} s_{1}(t)+\sqrt{1-\lambda} s_{0}(t)+e(t) \\
& H_{0}: y(t)=s_{0}(t)+e(t)
\end{aligned}
$$

$s_{1}(t)$ : Known signal. 
$s_{0}(t):$ Known noise.

$e(t)$ : Observation noise which is $N\left(0, \sigma^{2}\right)$.

$\lambda$ : Constant between 0 and 1.

$\mathrm{s}_{1}$ and $\mathrm{s}_{0}$ are uncorrelated, i.e. $\left\langle s_{1}^{*}(t) s_{0}(t)\right\rangle \approx \frac{1}{T}\left(\mathbf{s}_{1} \cdot \mathbf{s}_{0}\right)=0$, and have same PSD. By the Parseval theory [7], we can define

$$
\mathrm{s}_{1}^{T} \mathrm{~s}_{1}=\mathrm{s}_{0}^{T} \mathrm{~s}_{0}=E
$$

Using the result of Equation 13 we get:

$$
\begin{aligned}
& H_{1} \\
& \mathbf{y}^{T}\left\{\sqrt{\lambda} \mathbf{s}_{1}-(1-\sqrt{1-\lambda}) \mathbf{s}_{0}\right\} \underset{<}{<} \sigma^{2} \log \eta \\
& H_{0}
\end{aligned}
$$

y could be any signal between case $H_{1}$ and $H_{0}$. Place y under the two hypotheses $H_{1}$ and $H_{0}$, correspondingly we get the lower and upper bound of the threshold:

$$
\gamma_{0} \stackrel{\text { def }}{=}-(1-\sqrt{1-\lambda}) E \leq \sigma^{2} \log \eta \leq(1-\sqrt{1-\lambda}) E \stackrel{\text { def }}{=} \gamma_{1}
$$

and

$$
\Delta \gamma \stackrel{\text { def }}{=} \gamma_{1}-\gamma_{0}=2(1-\sqrt{1-\lambda}) E
$$

From Equation 9 we can see that as the SNR decreases, the $\lambda$ becomes smaller, therefore the $\Delta \gamma$ gets narrower which makes detection more difficult to do. Since the observation y could be any distribution, it is hard to find a sufficient statistic. And a theoretical calculation of $P_{D}$ and $P_{F}$ is difficult. However you always can do experiments to plot them. 


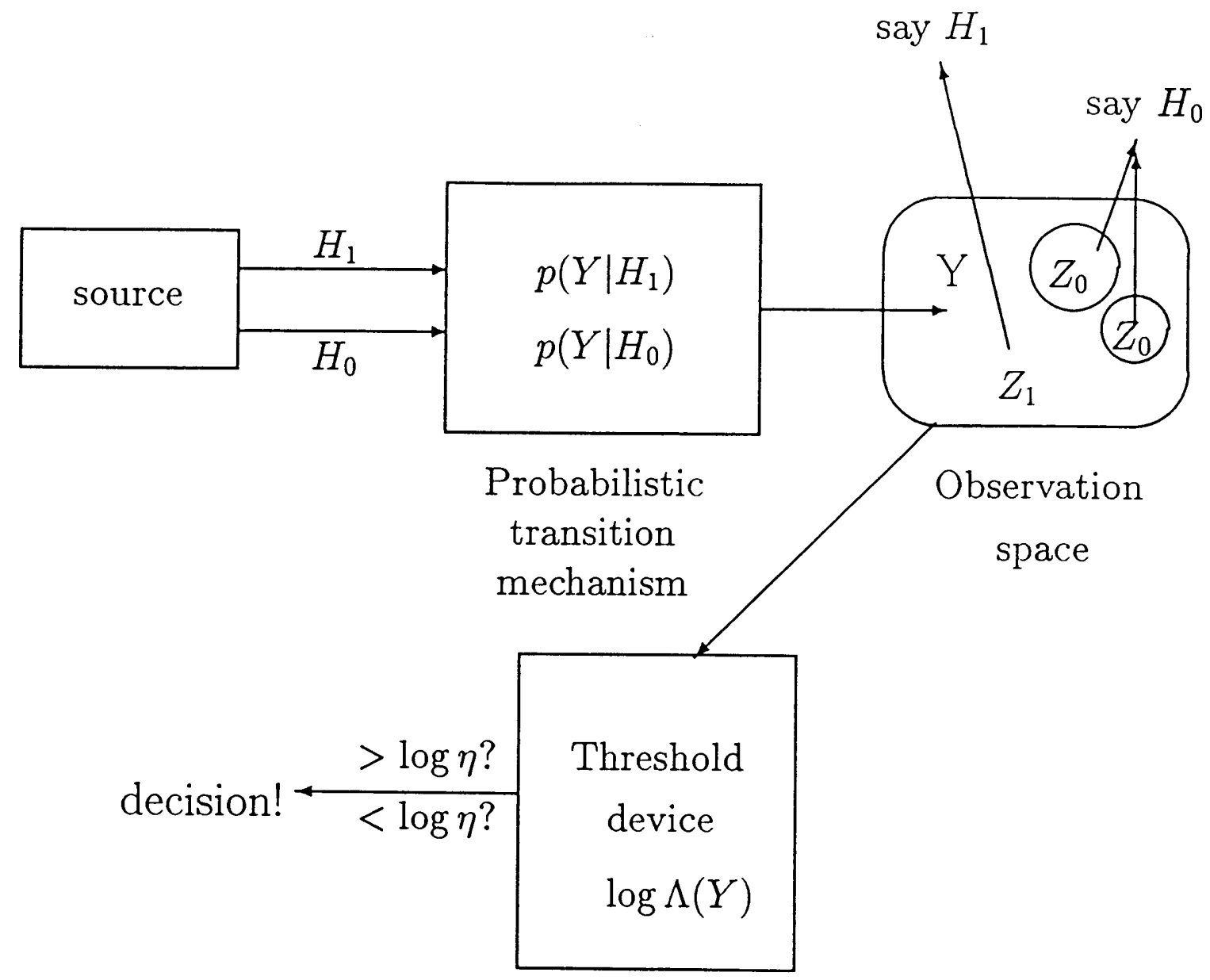

Figure 1. Components of a decision theory problem. 
(a)
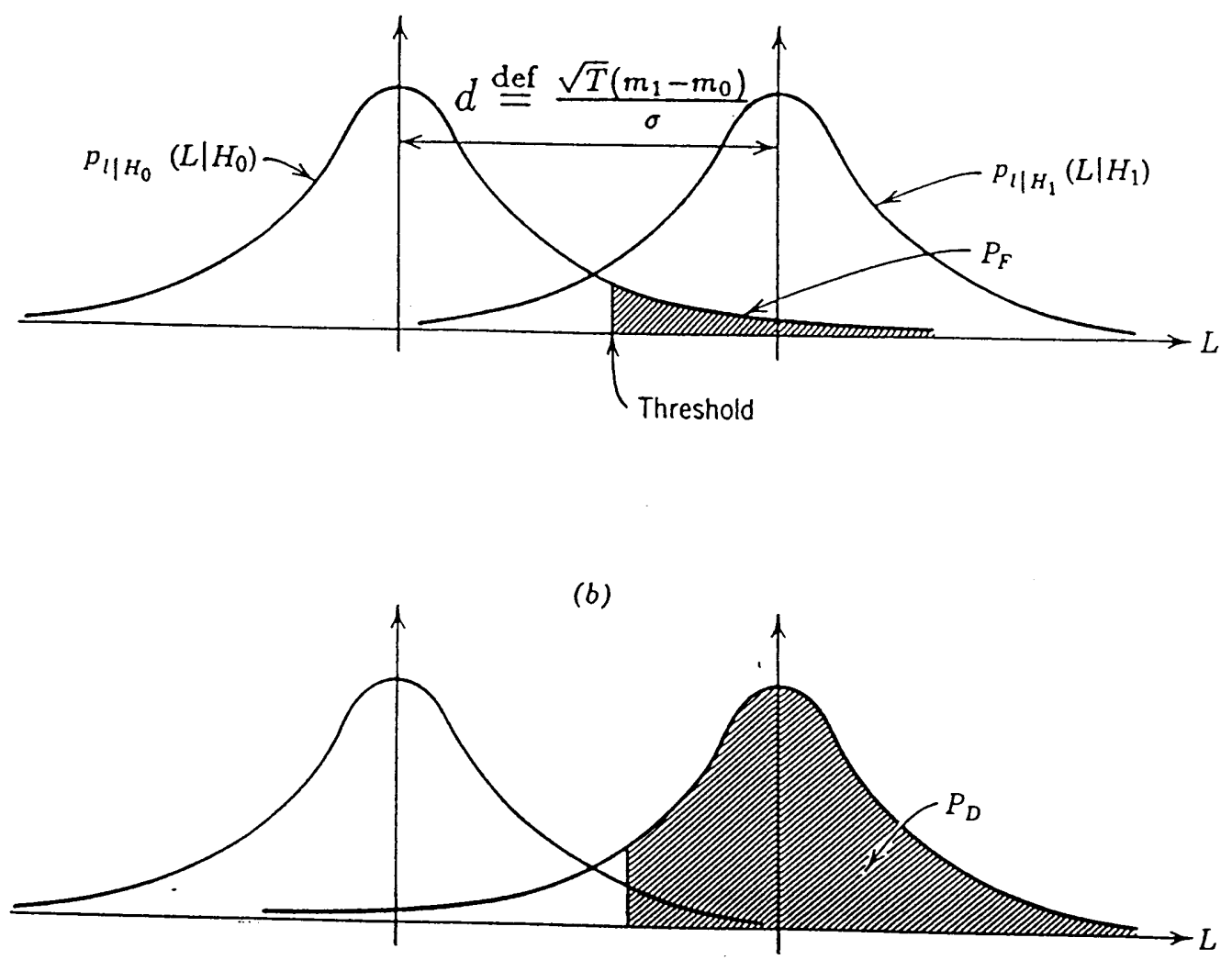

Figure 2. Error probabilities: (a) $P_{F}$ calculation, (b) $P_{D}$ calculation. 

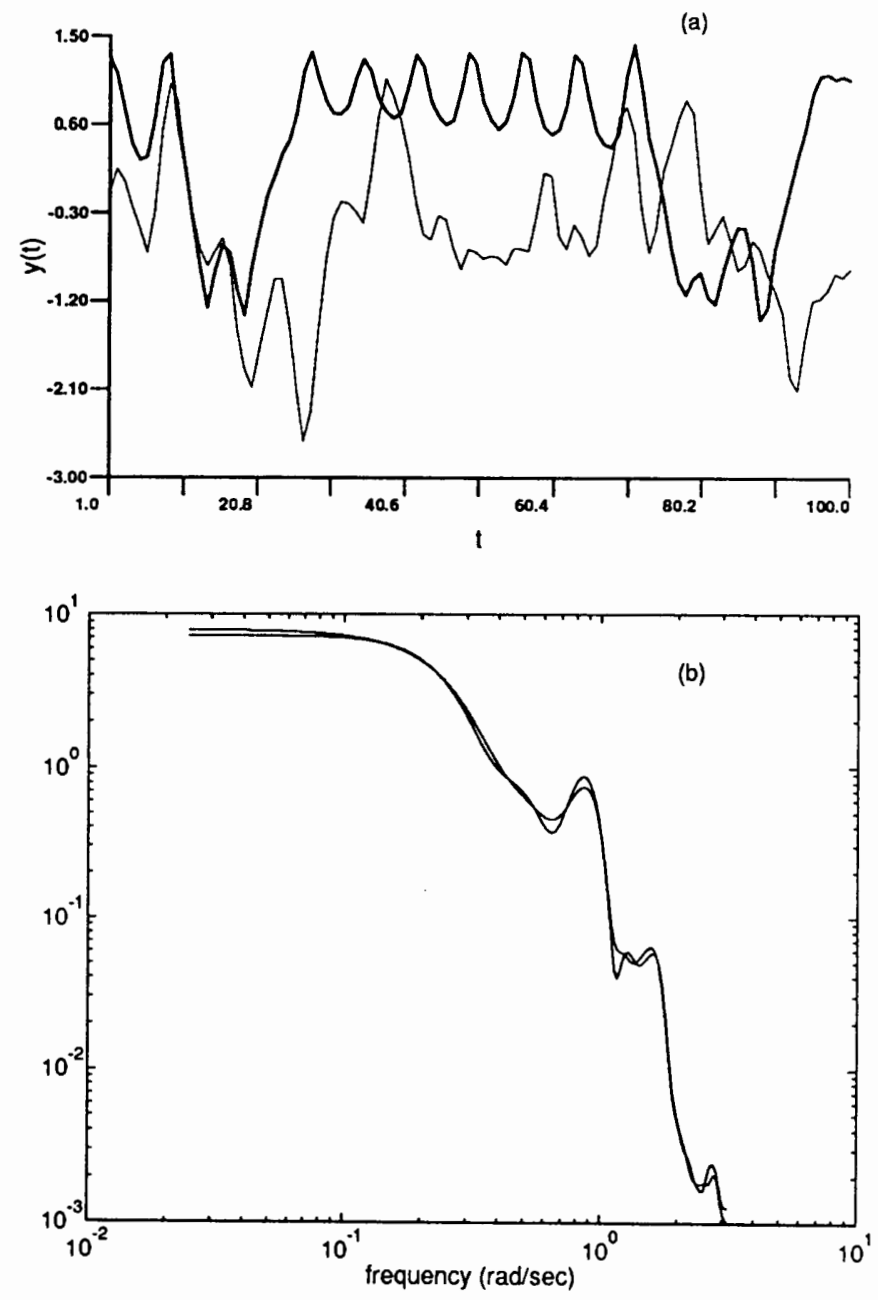

Figure 3. (a) Signals: the Duffing System (thick), a 15th order AR noise (thin), (b) PSD of the signal and noise (seen from the plot that they are very similar). 


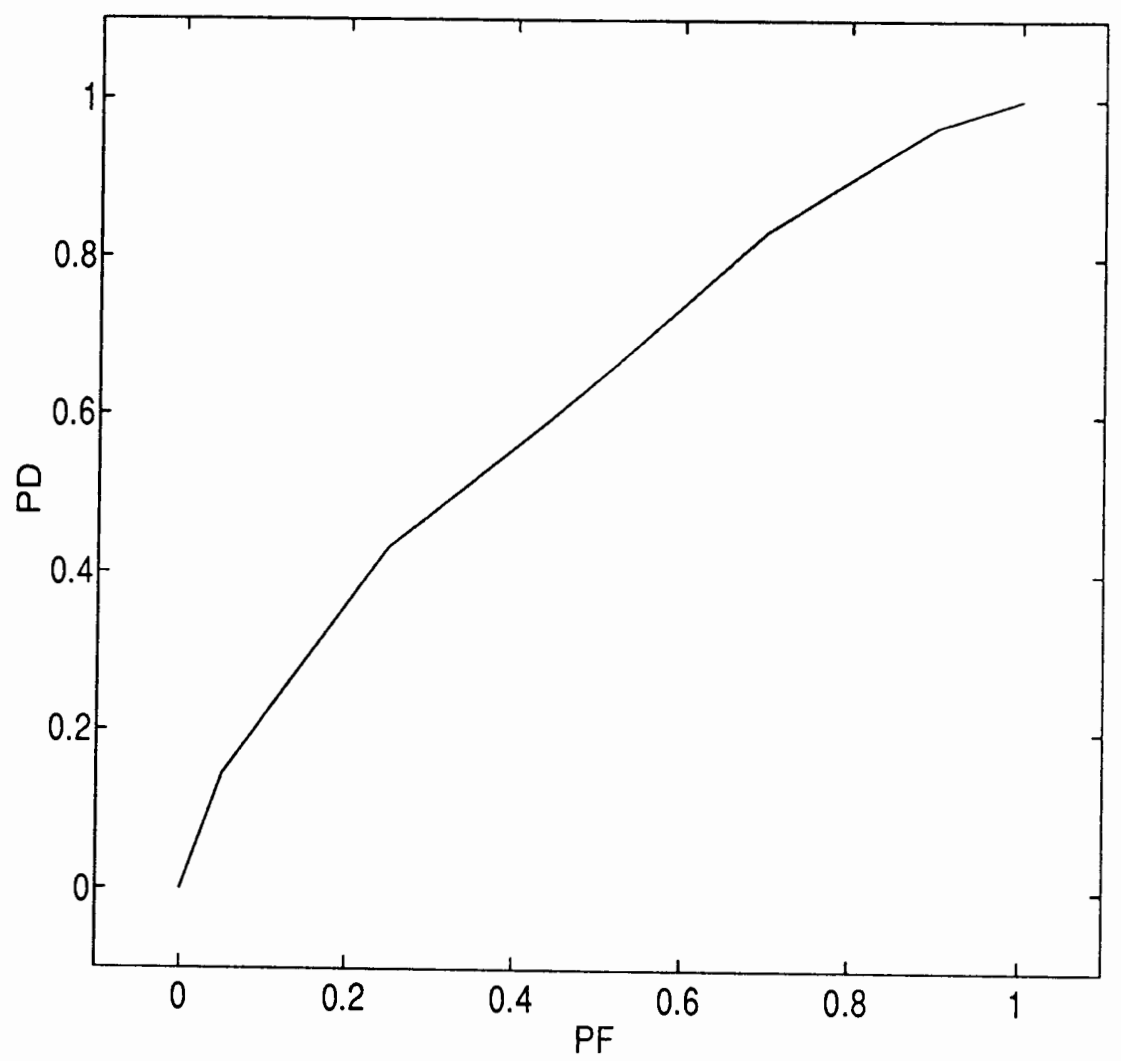

Figure 4. Experimental ROC for detecting the pure Duffing signal from the 15 th order AR noise using 10th order AR models. 


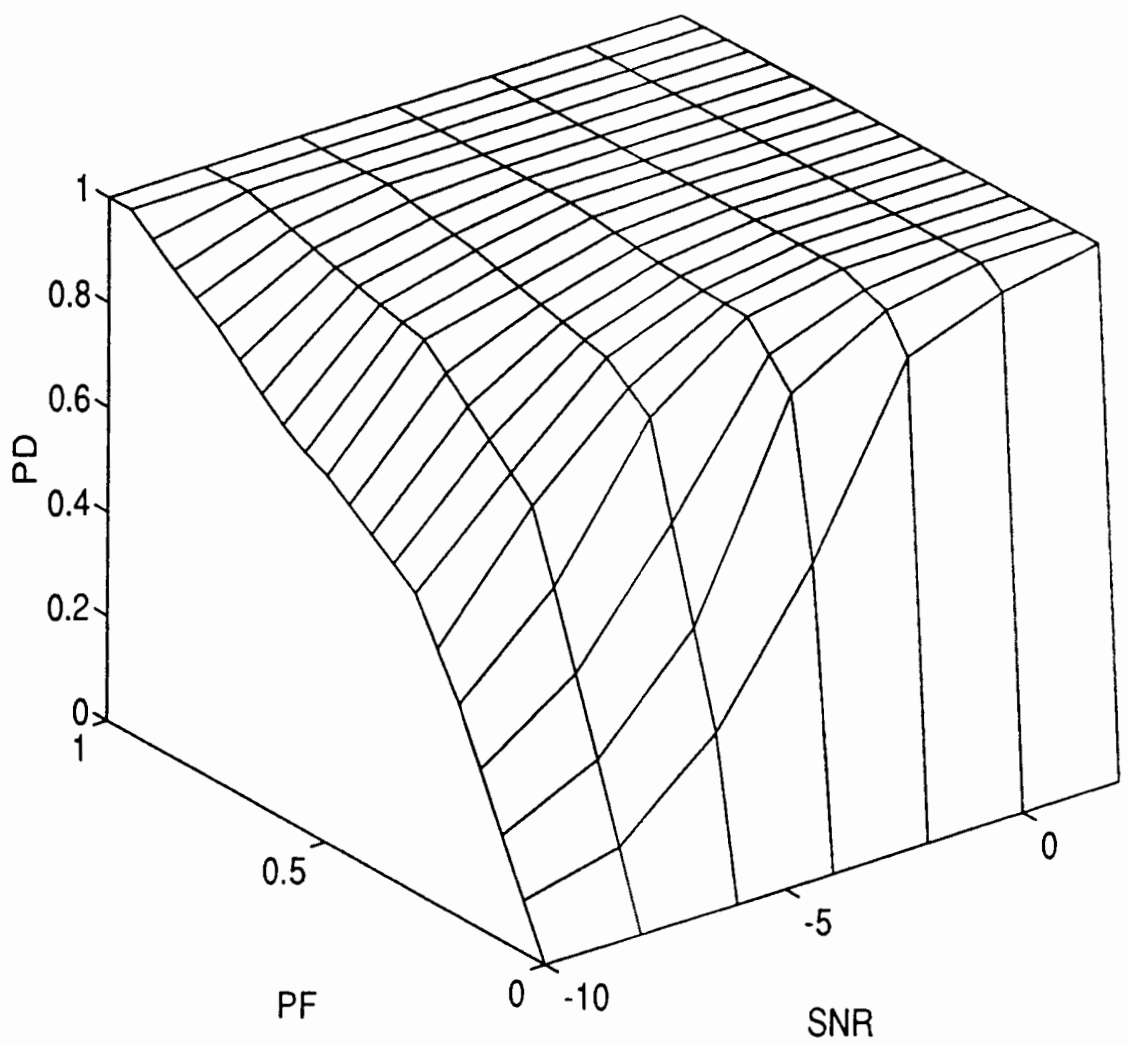

Figure 5. Experimental ROCs for detecting the noisy Duffing signal in 15 th order AR noise by using 30th order AR models. 


\section{CHAPTER III}

\section{SIMPLE HISTOGRAM MODEL}

\section{III.1 INTRODUCTION}

One is typically required to make a decision between low SNR signal and noise. So the model we design must be very sensitive to a signal. The AR model, as well as other linear models has failed to distinguish the difference between the signal and noise having similar second-order statistics. In order to deal with this intrinsically nonlinear problem, we introduce a simple Histogram Model (HM) which can outperform AR models in this type of detection.

The idea of using a Histogram Model in detection was inspired by Shaw [8], who used histograms to describe system dynamics. We use Markov chains for calculating $p\left(y_{1}^{T} \mid \theta\right)$ and high dimensional HMs to estimate the transition probabilities. One dimensional HM was considered as i.i.d. (independently identically distributed). From the plots of $y(t)$ vs. $y(t+1)$ shown in Figure 6 we can see a completely different dynamics between signal and noise. Numerical simulations showed flawless experimental ROCs at SNRs as small as -16db.

The advantage of this model is its simplicity, generality and ease of training. However, this intuitive model requires a large number of parameters which grow exponentially with the dimension. Compared to HFHMMs, the detection performance using this model is poorer and harder to improve upon. However, it is still a model worth studying in the sense of its superiority to linear models in dealing with 
chaotic aspects.

\section{III.2 1D HISTOGRAM MODEL}

We begin with a $1 \mathrm{D}$ (one dimensional) model. The noise corrupted signal is defined as before:

$$
\begin{gathered}
y(t)=\sqrt{\lambda} s_{1}(t)+\sqrt{1-\lambda} s_{0}(t) \\
S N R=10 \log _{10} \frac{1-\lambda}{\lambda},
\end{gathered}
$$

so that it has the similar PSD with the 15 th order AR noise. Figure 7 shows the histograms of the pure signal and noise, and Figure 8 plots a series of histograms of noisy signals with different SNRs. We can see from the figures that as SNR decreases, noise gradually dominates the corrupted signal whose distribution becomes closer to that of noise. At some values ( $\mathrm{SNR}$ at $-30 \mathrm{db}$ ) it is hard to distinguish them by visual inspection.

For $\mathbf{y}=\sqrt{\lambda} \mathbf{s}_{1}+\sqrt{1-\lambda} \mathbf{s}_{0}$, if $p_{s_{0}}\left(\mathbf{s}_{0}\right)$ and $p_{s_{1}}\left(\mathbf{s}_{1}\right)$ are known, $\mathrm{s}_{0}$ and $\mathbf{s}_{1}$ are uncorrelated, and we can calculate $p_{y}(\mathbf{y})$ in theory as

$$
p_{y}(\mathbf{y})=\frac{1}{\sqrt{\lambda}} \int_{-\infty}^{\infty} p_{s_{1}}\left(\frac{\mathbf{y}-\sqrt{1-\lambda} \mathbf{s}_{0}}{\sqrt{\lambda}}\right) p_{s_{0}}\left(\mathbf{s}_{0}\right) d \mathbf{s}_{0}
$$

And we can draw the same conclusion as indicated in the figures, i.e., as $\lambda$ decreases, the distribution of $\mathbf{y}$ is closer to that of the AR noise. An obvious example in this case is when $s_{1}$ and $s_{0}$ are two Gaussian distributions of $N\left(m_{1}, \sigma_{1}^{2}\right)$ and $N\left(m_{0}, \sigma_{0}^{2}\right)$ respectively. Then $\mathrm{y}$ is $N\left(m_{y}, \sigma_{y}^{2}\right)$. where

$$
\begin{aligned}
m_{y} & =\sqrt{\lambda} m_{1}+\sqrt{1-\lambda} m_{0} \\
\sigma_{y}^{2} & =\lambda \sigma_{1}^{2}+(1-\lambda) \sigma_{0}^{2}
\end{aligned}
$$


To return to our detection problem, first we used one sample sequence of observation $y$ to train and obtained parameters from a histogram. Then different sample sequences in $\mathrm{y}$ were used to do the detection test. Suppose $y(t)$ is i.i.d., using 1D HM to calculate $p(y \mid \theta)$ is just searching in the histogram table for the closest element to $y(t)$, then choosing its PDF to be $p(y(t) \mid \theta)$. So

$$
\begin{aligned}
& p\left(y_{1}^{T} \mid \theta_{1}\right)=\prod_{t=1}^{T} p\left(y(t) \mid \theta_{1}\right) \\
& p\left(y_{1}^{T} \mid \theta_{0}\right)=\prod_{t=1}^{T} p\left(y(t) \mid \theta_{0}\right)
\end{aligned}
$$

the LRT is

$$
\sum_{t=1}^{T} \log \frac{p\left(y(t) \mid \theta_{1}\right)}{p\left(y(t) \mid \theta_{0}\right)} \stackrel{\begin{array}{l}
H_{1} \\
H_{0}
\end{array}}{>} \log \eta
$$

Because of the logarithm in the calculation, we use $1 \times 10^{-15}$ instead of zero in the conditional PDF. The performance of detection in 1D HM is shown in Figure 9. We tried to use more bins to improve the performance, but the results are not as good as expected.

\section{III.3 2D HISTOGRAM MODEL}

The 2D (two dimensional) HM is a little bit more complicated. First the histogram should represent a joint PDF, i.e. $p(y(t), y(t+1))$. The joint PDF of pure signal and noise are plotted in Figure 10. We can see they are quite different. As SNR decreases, it is more and more difficult to see the difference between the noisy signal and noise only. See Figure 11.

Second, we can no longer treat $y(t)$ as i.i.d. We use a first order Markov 
chain and conditional statistics [9] to determine:

$$
p\left(y_{1}^{T} \mid \theta\right)=p(y(1) \mid \theta) \prod_{t=2}^{T} p(y(t) \mid y(t-1), \theta)
$$

where

$$
p(y(t) \mid y(t-1), \theta)=\frac{p(y(t-1), y(t) \mid \theta)}{p(y(t-1) \mid \theta)}
$$

The LRT is

$$
\log \frac{p\left(y(1) \mid \theta_{1}\right)}{p\left(y(1) \mid \theta_{0}\right)}+\sum_{t=2}^{T} \log \frac{p\left(y(t) \mid y(t-1), \theta_{1}\right)}{p\left(y(t) \mid y(t-1), \theta_{0}\right)} \underset{H_{0}}{<} \log \eta
$$

The detection performance using a 2D HM is shown in Figure 12, and is better than that using a 1D HM. If we use a longer sequence to train, performance should be improved even more.

\section{III.4 HIGH DIMENSIONAL HISTOGRAM MODEL}

The joint PDF of higher dimensions is hard to plot because it involves a figure with at least four dimensions. To simplify the display, we make a cross section, as shown in Figure 13 which is $p(y(t), y(t+1), y(t+2))$ of signal and noise cut at $y(t)=0.1$.

Similarly we can derive higher dimensional HMs by using higher order Markov chains instead of the first order, i.e.,

$$
p\left(y_{1}^{T} \mid \theta\right)=p\left(y_{1}^{D} \mid \theta\right) \prod_{t=D+1}^{T} p\left(y(t) \mid y_{t-D}^{t-1}, \theta\right)
$$

where

$$
p\left(y(t) \mid y_{t-D}^{t-1}, \theta\right)=\frac{p\left(y_{t-D}^{t} \mid \theta\right)}{p\left(y_{t-D}^{t-1} \mid \theta\right)}
$$


and the LRT is:

$$
\log \frac{p\left(y_{1}^{D} \mid \theta_{1}\right)}{p\left(y_{1}^{D} \mid \theta_{0}\right)}+\sum_{t=D+1}^{T} \log \frac{p\left(y(t) \mid y_{t-D}^{t-1}, \theta_{1}\right)}{p\left(y(t) \mid y_{t-D}^{t-1}, \theta_{0}\right)} \stackrel{\substack{H_{1} \\ H_{0}}}{>} \log \eta
$$

Experimental ROCs from a 3D (three dimensional) HM are shown in Figure 14. The performance is only a little better than that from a 1D HM. The reason for the poor performance is that, due to computational limitations we did not use sufficient data to train compared to the huge numbers of parameters in the model. i.e. if we use $20,000 \times 47$ long data sequence, detection will be better than that by using 2D HM.

The experiments indicate that to some degree (actually 47 bins in one dimension is enough) increasing bins does not help improve detection performance. It is a severe limitation of HM; however HFHMMs can improve the detection performance by increasing the number of parameters, as we discuss in later chapters. 

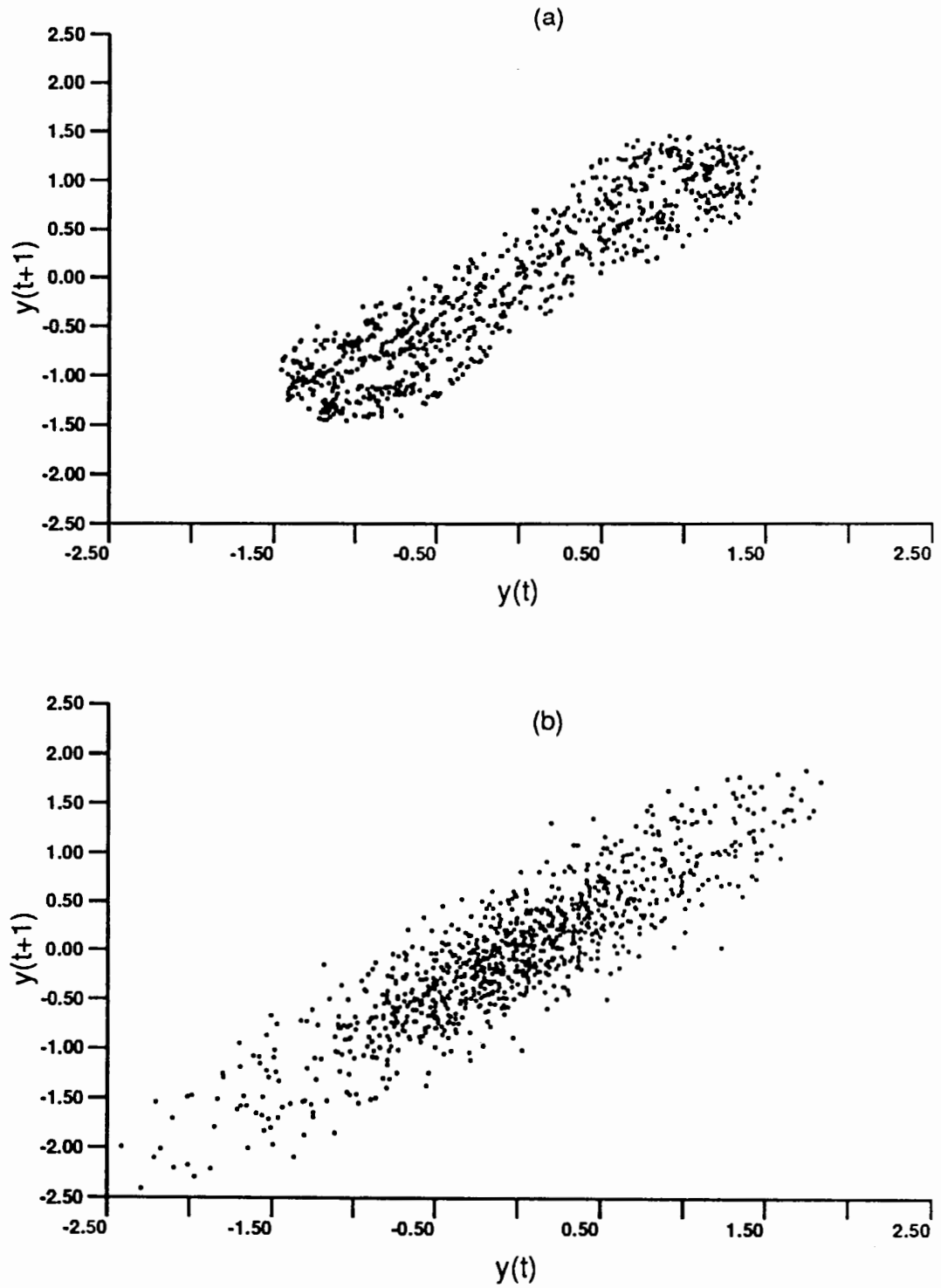

Figure 6. Plots of $y(t+1)$ vs. $y(t)$, (a) the Duffing signal, (b) the 15 th order AR time series. 

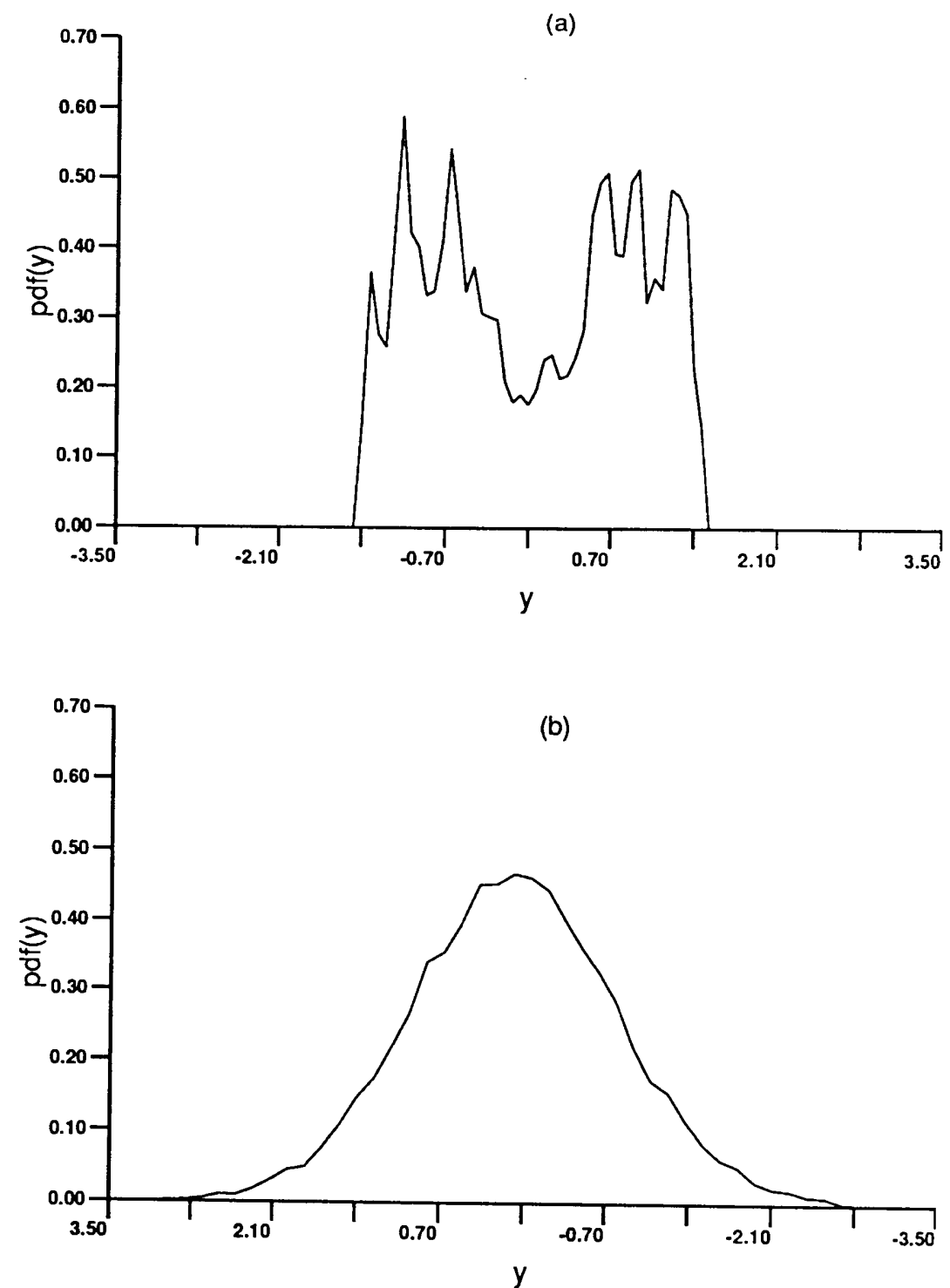

Figure 7. 1D histogram with 47 bins and 4,000 length of training sequence, (a) the Duffing signal, (b) the 15 th order AR noise. 

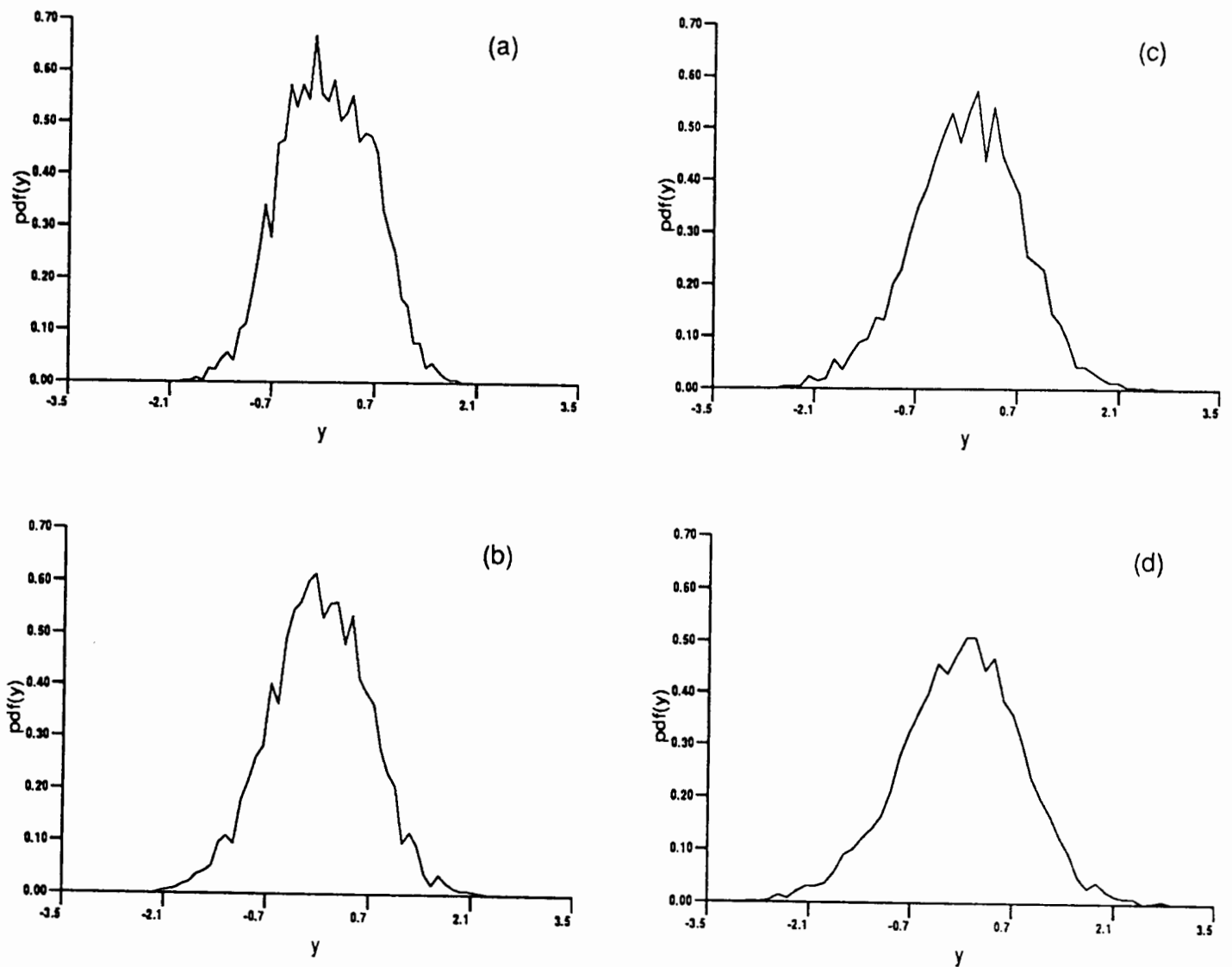

Figure 8. 1D histogram of mixed signal with 47 bins and 4,000 length of training sequence, (a) SNR $=0$, (b) SNR $=-9$, (c) SNR $=-17$, (d) SNR $=-30$. 


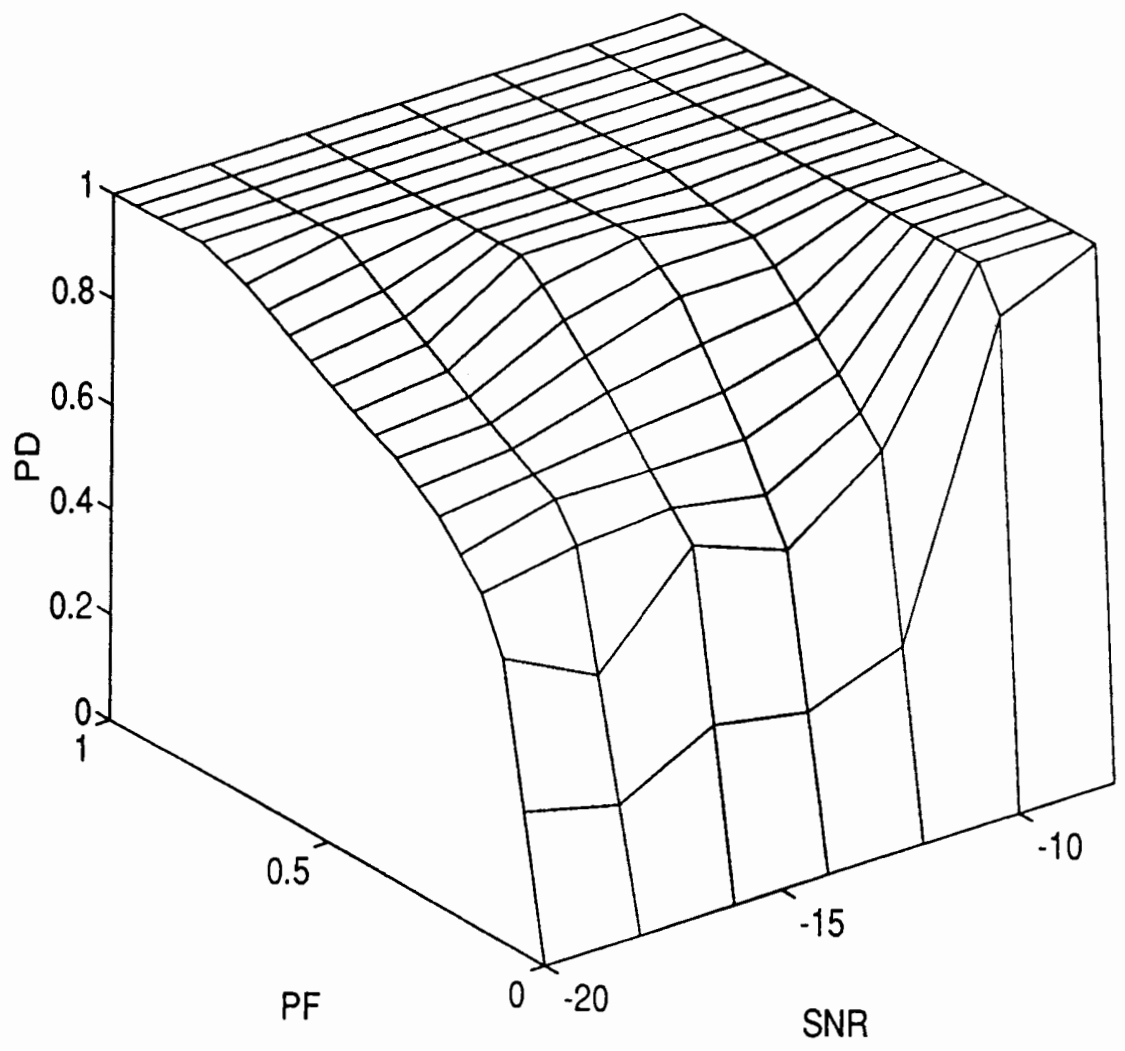

Figure 9. Experimental ROCs for detecting the noisy Duffing signal in 15th order AR noise using a 1D HM with 47 bins. The lengths of training and testing sequence are all 4,000. 

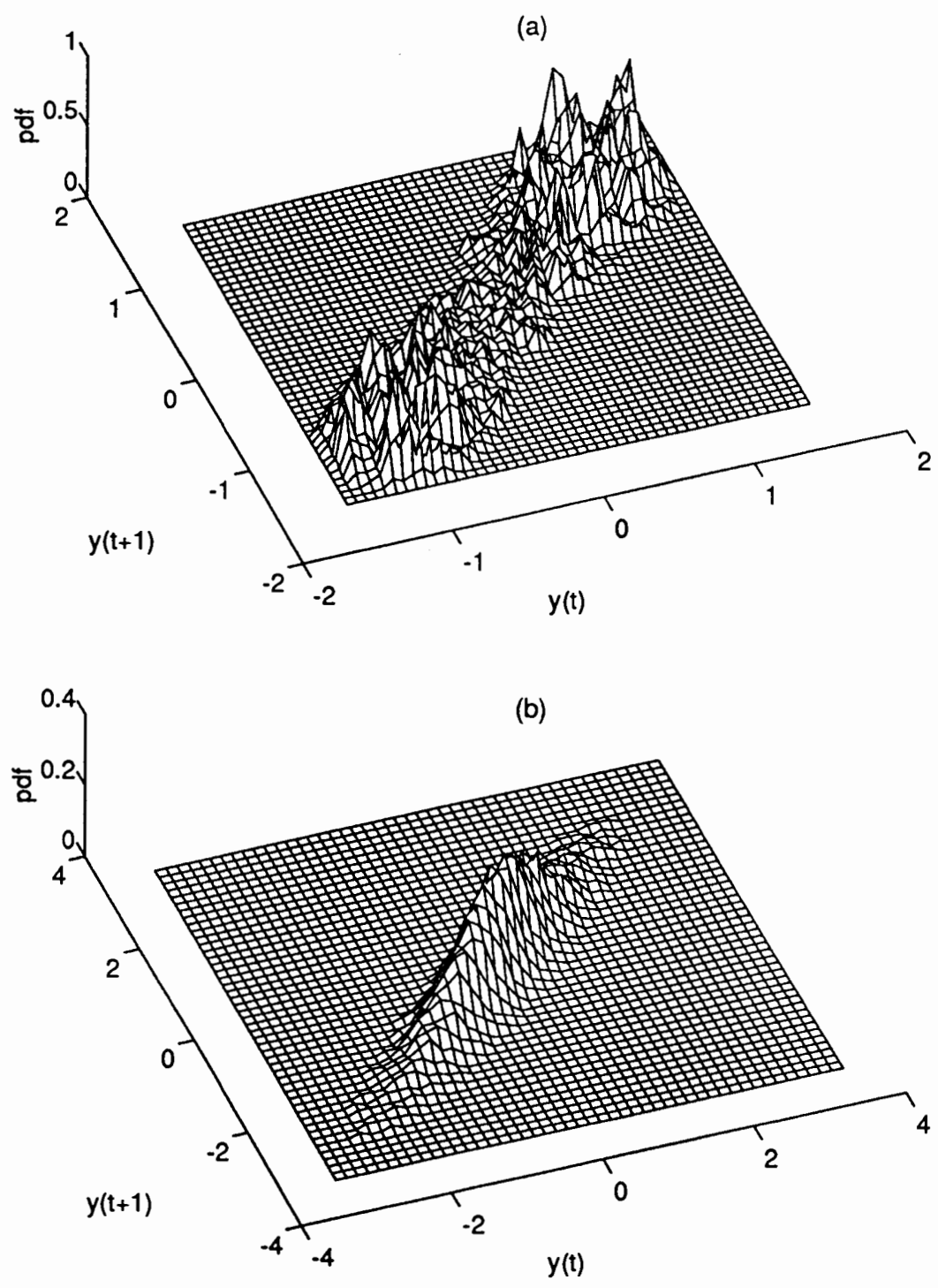

Figure 10.2D histogram with $47^{2}$ bins and training sequence of length 20,000 . (a) the Duffing signal, (b) the I5th order AR noise. 

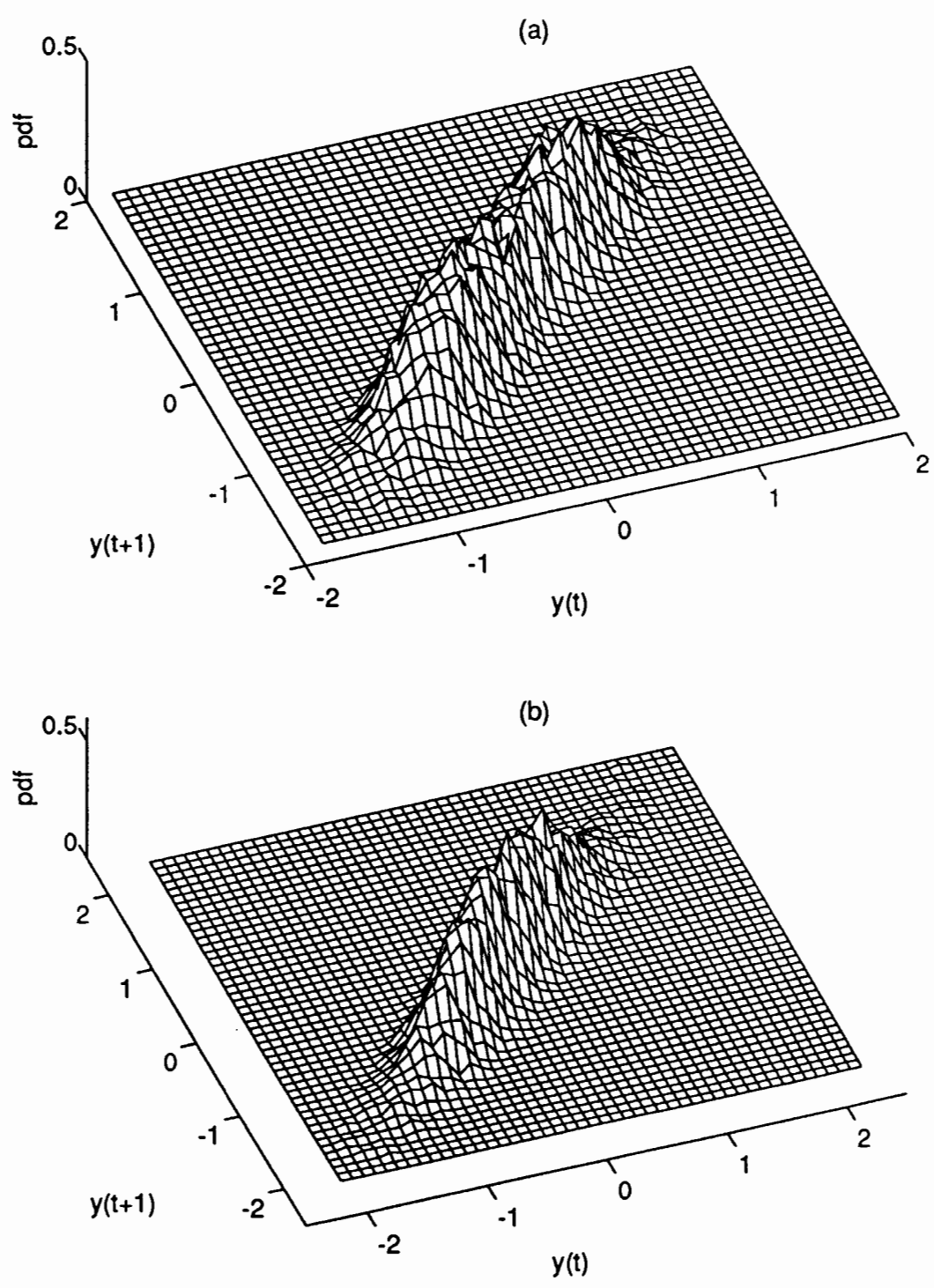

Figure 11. 2D histogram of the noisy Duffing signal with $47^{2}$ bins and training sequence of length 20,000 . (a) $\mathrm{SNR}=6$, (b) SNR $=0$. 


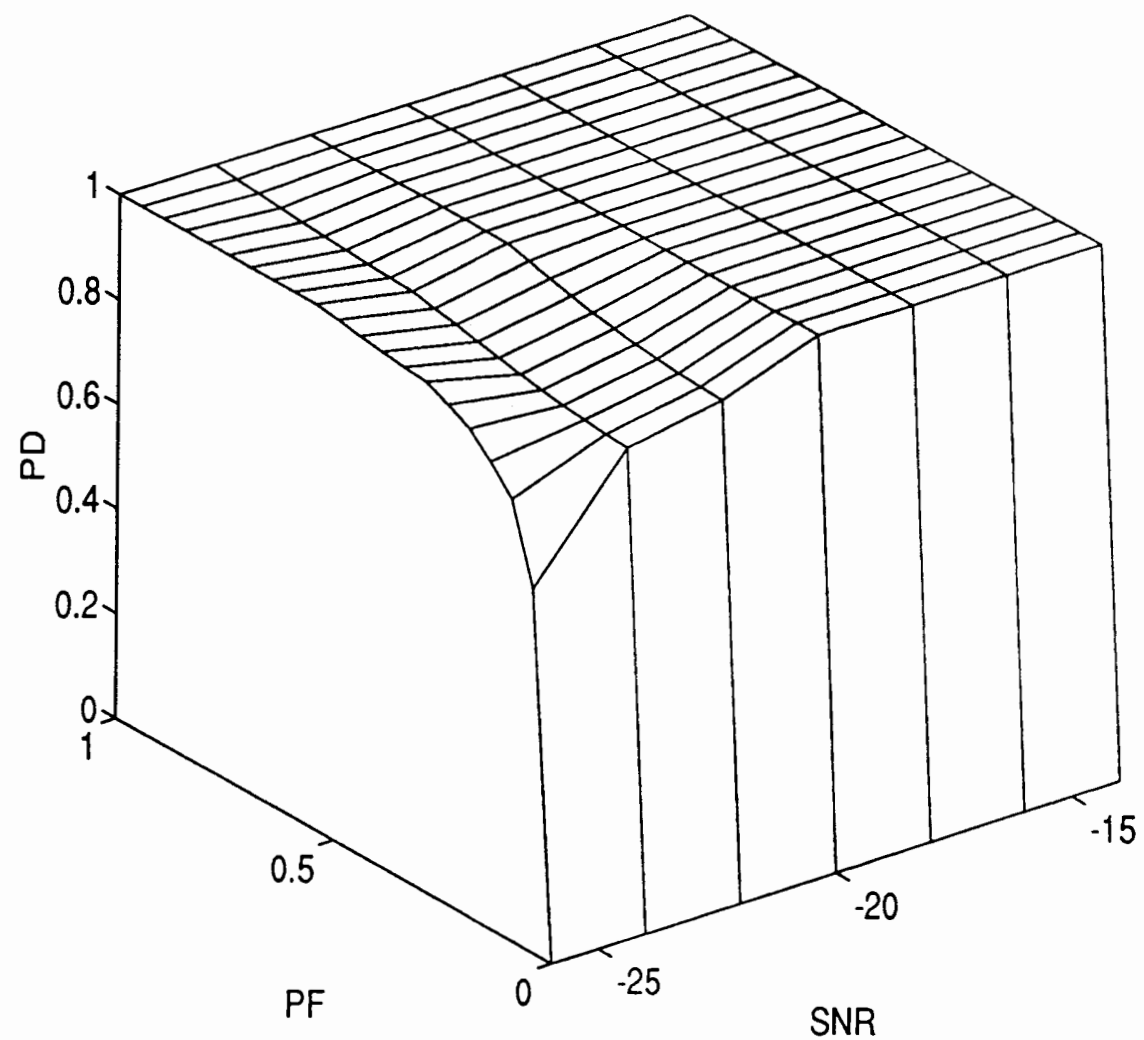

Figure 12. Experimental ROCs for detecting the noisy Duffing signal in 15th order AR noise using 2D HM, where $47^{2}$ bins are used, the length of training and testing sequence is 20,000 and 4,000 respectively. 

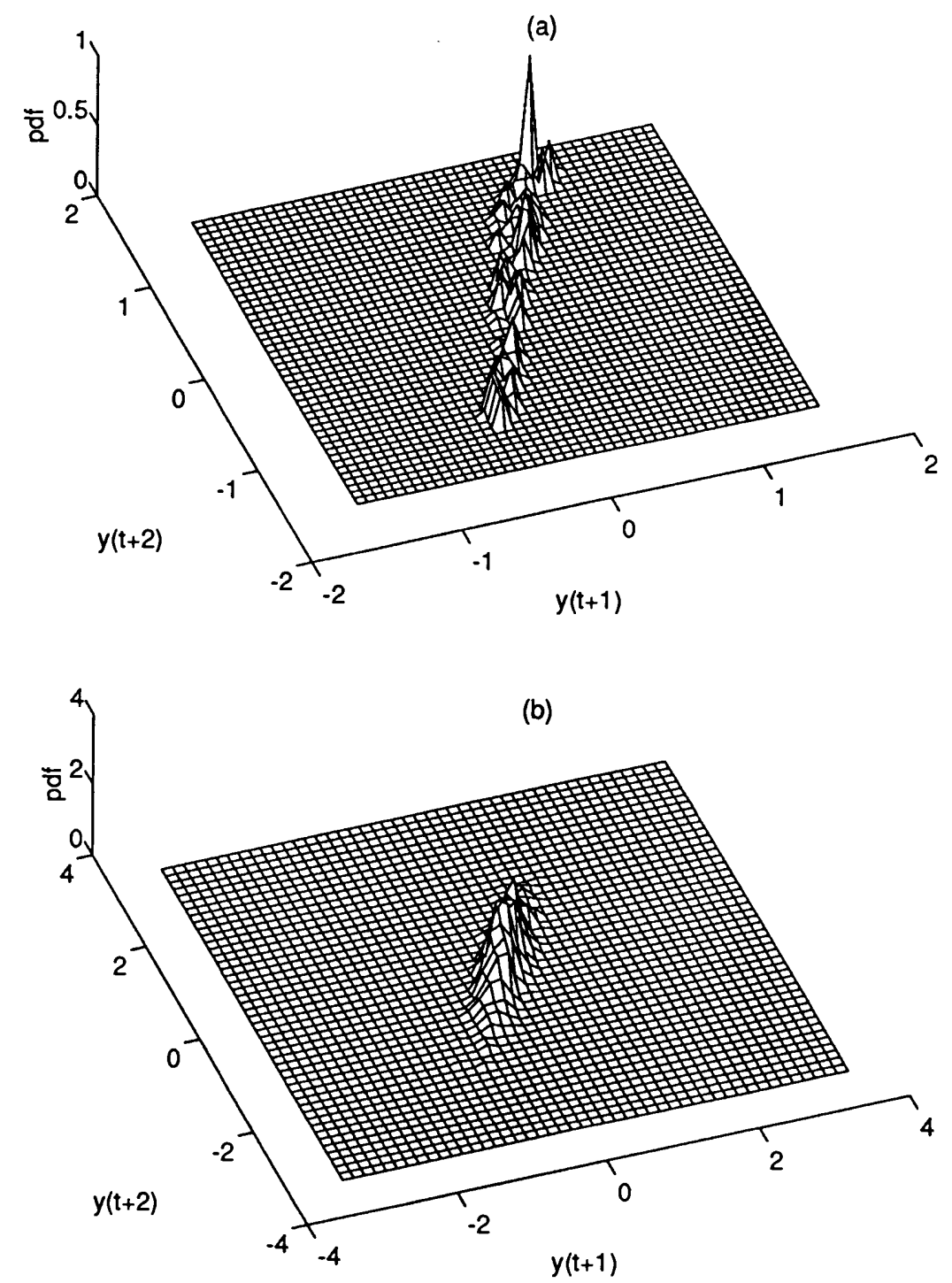

Figure 13. 3D histogram with $47^{3}$ bins and training sequence of length 20,000 . Cut at $y(t)=0.1$, (a) the Duffing signal, (b) the 15th order AR noise. 


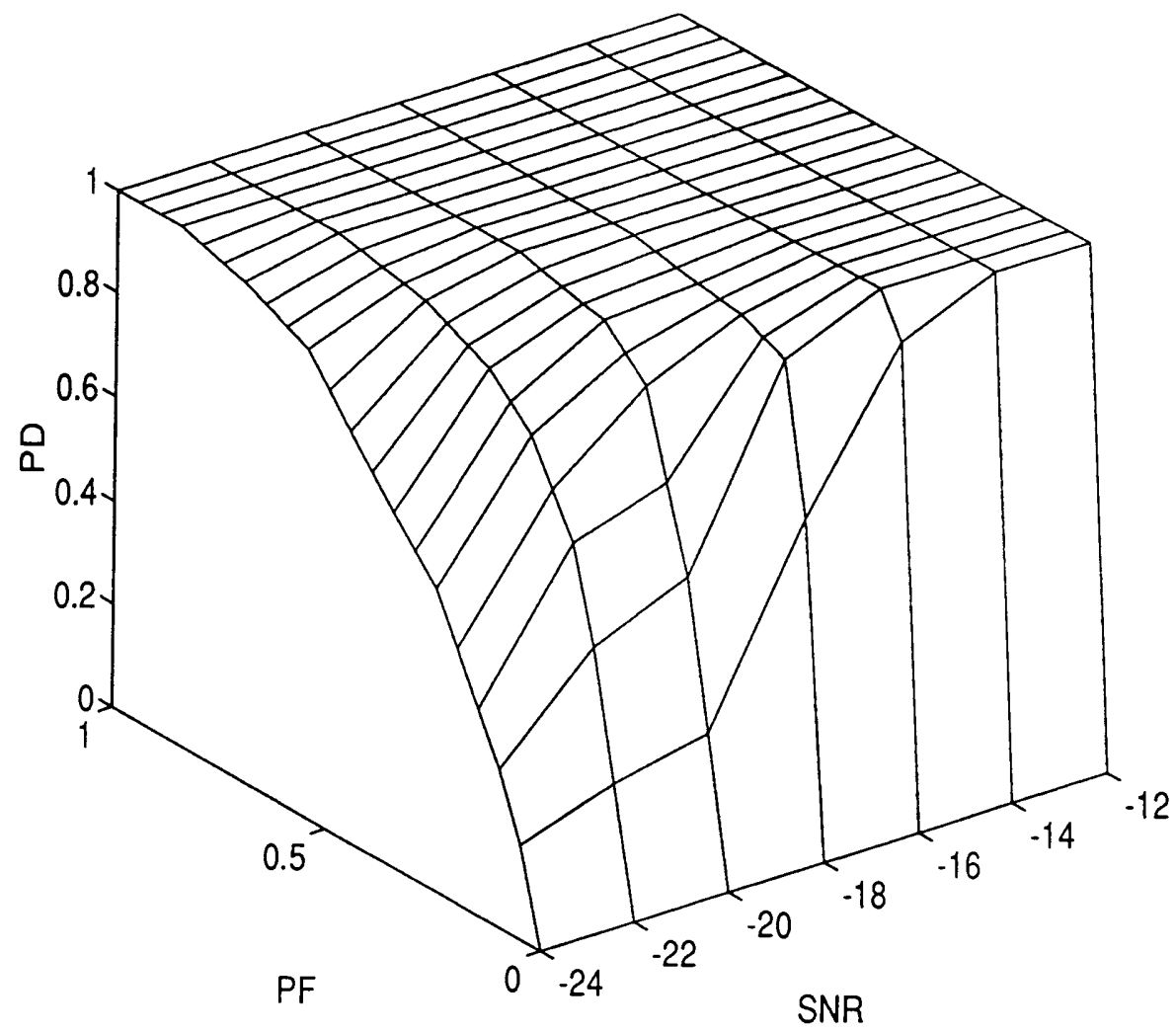

Figure 14. Experimental ROCs for detecting the noisy Duffing signal in 15th order AR noise using $3 \mathrm{D} \mathrm{HM}$, where $47^{3}$ bins are used, the length of training and testing sequence is 20,000 and 4,000 respectively. 


\section{CHAPTER IV}

\section{HIDDEN FILTER HIDDEN MARKOV MODEL 1}

\section{IV.1 INTRODUCTION}

Hidden Markov Modeling (HMM) is a probabilistic technique for the study of discrete time series. Recently HMM has been increasingly attractive in language, speech and signal processing. Detection [10] by using HMMs is just one of its applications.

In Poritz [1] the two components of the model structure are summarized as a hidden Markov chain and a finite set of output probability distributions. The main tool in HMM is the Baum-Welch (forward-backward) algorithm for maximum likelihood estimation of the model parameters. To work on HFHMMs, we used variations of the programs written by Fraser and Dimitriadis [2]. The models are based on several hidden states, such that in a particular state there is a scalar output which has a Gaussian Distribution whose mean is a linear AR function of previous samples.

This chapter attempts to bridge the gap between dynamic systems and signal processing by applying the HFHMM to the chaotic signal detection problem. Our approach is to develop methods to build self-adaptive models that can exploit the chaotic aspects of signals. Such models are very important in detection and other applications. 


\section{IV.2 MODELING}

This section draws mainly from Fraser and Dimitriadis [2].

\section{IV.2.1 Model Structure}

As described above, the key to building a HMM is designing the unobserved Markov chain and deciding what the output probability distributions should be. The two components are shown in Figure 15 where $s_{o}$ and $s_{i}$ represent hidden states, and $y(t)$ stands for the scalar output which depends on the states. The model parameters in HFHMMs are:

- $P_{s(t+1) \mid s(t)}\left(s_{i}, s_{o}\right)$ denotes the unobserved first order Markov chain.

- $P_{y(t) \mid s(t), \mathbf{x}(t)}\left(y, s_{i}, \mathbf{x}\right)=\frac{1}{\sqrt{2 \pi} \sigma_{s_{i}}} \exp -\frac{\left(y-\hat{y}_{s_{i}}\right)^{2}}{2 \sigma_{s_{i}}^{2}}$ is the Gaussian output distribution with mean of AR function

$$
\hat{y}_{s_{i}}=\bar{y}_{s_{i}}+\mathbf{a}_{s_{i}} \cdot \mathbf{x}
$$

In the state space view, observations are functions of internal states. It can be described as the equations

$$
\begin{aligned}
x(t+1) & =F(x(t))+\eta_{1}(t+1) \\
y(t) & =G(x(t))+\eta_{2}(t)
\end{aligned}
$$

where $x$ is a point in an unobserved vector state space, in HFHMM it consists of the history vector $\mathbf{x}(t)$ and the hidden state $s(t) . F$ describes the state space dynamics, $y$ is an observation (in this thesis the observations are scalars) derived from the state by the function $G$, and $\eta_{1}$ and $\eta_{2}$ describe the dynamical noise and observation noise respectively. In a detection application, the state space variables are integrated out 
to give the required maps from sequences of observation to likelihood. For the noise free measurements of a chaotic (low dimensional deterministic aperiodic) process, $\eta_{1}$ and $\eta_{2}$ are zeros, and $\mathbf{x}(t)=y_{t-D}^{t}$. Of course, the inevitable noise makes things complicated. Specifically, noise will make the choice of coordinates for the state space important [11] [12] [13].

The simplest HMMs are discretized versions of the state space view of time series (Eqn. 15) having discrete states and discrete outputs. In principle, given enough hidden states and output levels, such a discrete model could describe Equation 15 arbitrarily well.

\section{IV.2.2 The EM algorithm}

The EM algorithm [14] adjusts model parameters $\theta$ to maximize the likelihood of observations $\mathbf{y}$. It operates on a class of models which includes an unobserved sequence of discrete states $q, P_{\mathbf{y}, q, \theta}$. The steps in the algorithm are:

1. Guess a initial value of $\theta$

2. Choose $\hat{\theta}$ to maximize $\left\langle\log P_{\mathbf{y}, q, \hat{\theta}}(\mathbf{y}, q)\right\rangle_{q \mid \mathbf{y}, \theta}$

3. Set $\theta=\hat{\theta}$.

4. If not converged, go to 2 .

From [15] we can show that the above procedure is as same as maximizing $P_{\theta}(\mathbf{y})$ :

$$
\begin{gathered}
P_{\mathbf{y}, \hat{\theta}}(\mathbf{y})=\frac{P_{q, \mathbf{y}, \hat{\theta}}(q, \mathbf{y})}{P_{q \mid \mathbf{y}, \hat{\theta}}(q, \mathbf{y})} \\
\log P_{\mathbf{y}, \hat{\theta}}(\mathbf{y})=\log P_{q, \mathbf{y}, \hat{\theta}}(q, \mathbf{y})-\log P_{q \mid \mathbf{y}, \hat{\theta}}(q, \mathbf{y})
\end{gathered}
$$

Note $\left\langle\log P_{\mathbf{y}, \hat{\theta}}(\mathbf{y})\right\rangle_{q \mid \mathbf{y}, \theta}=\log P_{\mathbf{y}, \hat{\theta}}(\mathbf{y})$ because $q$ does not appear inside the \langle\rangle$_{q \mid \mathbf{y}, \theta}$. So

$$
\log P_{\mathbf{y}, \hat{\theta}}(\mathbf{y})=\left\langle\log P_{q, \mathbf{y}, \hat{\theta}}(q, \mathbf{y})\right\rangle_{q \mid \mathbf{y}, \theta}-\left\langle\log P_{q \mid \mathbf{y}, \hat{\theta}}(q, \mathbf{y})\right\rangle_{q \mid \mathbf{y}, \theta}
$$


Using Gibbs inequality for two distributions $P$ and $Q$ which says

$$
\sum_{x} P(x) \log \frac{Q(x)}{P(x)} \leq 0
$$

we get

$$
\left\langle\log P_{q \mid \mathbf{y}, \hat{\theta}}(q, \mathbf{y})\right\rangle_{q \mid \mathbf{y}, \theta} \leq\left\langle\log P_{q \mid \mathbf{y}, \theta}(q, \mathbf{y})\right\rangle_{q \mid \mathbf{y}, \theta}
$$

Thus if $\hat{\theta}$ is chosen so that

$$
\left\langle\log P_{q, \mathbf{y}, \hat{\theta}}(q, \mathbf{y})\right\rangle_{q \mid \mathbf{y}, \theta} \geq\left\langle\log P_{q, \mathbf{y}, \theta}(q, \mathbf{y})\right\rangle_{q \mid \mathbf{y}, \theta}
$$

Equation 16 yields

$$
P_{\mathbf{y}, \hat{\theta}}(\mathbf{y}) \geq P_{\mathbf{y}, \theta}(\mathbf{y})
$$

and the algorithm goes uphill.

\section{IV.2.3 HFHMMs}

As described before, HFHMMs consist of two parts, the hidden Markov chain $P_{s(t+1) \mid s(t)}\left(s_{i}, s_{j}\right)$ and the output distribution $P_{y(t) \mid s(t), \mathbf{x}(t)}\left(y, s_{i}, \mathbf{x}\right)$, where $s_{i} \in S$, $S=\left\{s_{1}, s_{2}, \ldots, s_{N s}\right\}$. The entire model parameters $\theta$ include all the parameters for all states. For each state $s$ the parameters are: the transition probabilities $P_{s(2) \mid s(1)}\left(s_{i}, s_{j}\right)$, constant part $\bar{y}_{s}$, AR coefficients $\mathrm{a}_{s}$ and standard deviation $\sigma_{s}$. Assume $\mathbf{x}(1)$ is available, the likelihood is

$$
P\left(y_{1}^{T}\right)=\sum_{q \in Q} P\left(y_{1}^{T}, q\right)
$$

where $Q$ denotes the set of all possible state sequences. The following manipulations show how the EM algorithm works in maximizing $P_{\theta}\left(y_{1}^{T}\right)$.

$$
\begin{gathered}
P\left(y_{1}^{T}, q\right)=P\left(y_{1}^{T} \mid q\right) P(q) \\
P\left(y_{1}^{T} \mid q\right)=P(y(1) \mid q) \prod_{t=2}^{T} P\left(y(t) \mid q, y_{1}^{t-1}\right)
\end{gathered}
$$

In HFHMMs a two step Markov hypotheses is made: 
1. The hidden process is first order Markov.

$$
P\left(s_{q}(t) \mid s_{q}(1), \ldots, s_{q}(t-1)\right)=P\left(s_{q}(t) \mid s_{q}(t-1)\right)
$$

so

$$
P(q)=P\left(s_{q}(1)\right) \prod_{t=2}^{T} P\left(s_{q}(t) \mid s_{q}(t-1)\right)
$$

2. The output process depends only on the current state and D previous outputs.

$$
P\left(y(t) \mid s_{1}^{t}, y_{1}^{t-1}\right)=P(y(t) \mid s(t), \mathrm{x}(t))
$$

so

$$
P\left(y_{1}^{T} \mid q\right)=\prod_{t=1}^{T} P\left(y(t) \mid s_{q}(t), \mathbf{x}(t)\right)
$$

Therefore Equation 19 becomes

$$
P\left(y_{1}^{T}, q\right)=P\left(y(1) \mid s_{q}(1), \mathbf{x}(1)\right) P\left(s_{q}(1)\right) \prod_{t=2}^{T} P\left(y(t) \mid s_{q}(t), \mathbf{x}(t)\right) P\left(s_{q}(t) \mid s_{q}(t-1)\right)
$$

Thus given $\mathbf{x}(\mathrm{t})$ and $s(t-1)$, earlier values of $s$ and $\mathbf{x}$ are irrelevant to current output, i.e.,

$$
\begin{aligned}
P\left(y(t), s(t) \mid y_{1}^{t-1}, s_{1}^{t-1}\right) & =P(y(t), s(t) \mid \mathbf{x}(t), s(t-1) \\
& =P(y(t) \mid s(t), \mathbf{x}(t)) P(s(t) \mid s(t-1))
\end{aligned}
$$

Equation 21 implies

$$
P(s(t) \mid s(t-1), \mathbf{x}(t))=P(s(t) \mid s(t-1))
$$

In the EM algorithm the expectation is with respect to the distribution $P_{\theta}\left(q \mid y_{1}^{T}\right)=$ $P_{\theta}\left(y_{1}^{T}, q\right) / P_{\theta}\left(y_{1}^{T}\right)$. If we let $w(q)=P_{\theta}\left(y_{1}^{T}, q\right)$ and $W=\sum_{q^{\prime} \in Q} w\left(q^{\prime}\right)$, so $P_{\theta}\left(q \mid y_{1}^{T}\right)=$ $w(q) / W$, the number of elements in $Q$ depends exponentially on $T$. The sum will 
be evaluated by FBA in the next subsection. Equation 20 and the EM algorithm lead to the expression for the required maximization:

$$
\begin{aligned}
W\left\langle\log P_{y_{1}^{T}, q, \hat{\theta}}\left(y_{1}^{T}, q\right)\right\rangle_{q \mid y_{1}^{T}, \theta}= & \sum_{q} w(q) P_{s(1)}(s(1)) \\
& +\sum_{q, t=1}^{T-1} w(q) \log P_{\hat{\theta}}\left(s_{q}(t+1) \mid s_{q}(t)\right) \\
& +\sum_{q, t=1}^{T} w(q) \log P\left(y(t) \mid s_{q}(t), \mathrm{x}(t)\right)
\end{aligned}
$$

and maximization of Equation 22 is equivalent to maximizing

$$
F_{1}(\hat{\theta}) \stackrel{\text { def }}{=} \sum_{q} v(q) P_{s(1)}(s(1))+\sum_{q, t=1}^{T-1} w(q) \log P_{\hat{\theta}}\left(s_{q}(t+1) \mid s_{q}(t)\right)
$$

and minimizing

$$
\begin{aligned}
F_{2}(\hat{\theta}) & \stackrel{\text { def }}{=} \sum_{q, t=1}^{T} w(q)\left\{\log \sigma_{s_{q(t)}}+\frac{\left(y(t)-\bar{y}_{s_{q(t)}}-\mathbf{a}_{s_{q(t)}} \cdot \mathbf{x}(t)\right)^{2}}{2 \sigma_{s_{q(t)}}^{2}}\right\} \\
& \stackrel{\text { def }}{=} \sum_{q, t} w(q) f_{2}\left(\hat{\theta}_{s_{q(t)}}, y(t), \mathbf{x}(t)\right) \\
& =\sum_{s, t}\left\{f_{2}\left(\hat{\theta}_{s_{q(t)}}, y(t), \mathbf{x}(t)\right) \sum_{q} w(q) \delta_{s, s_{q(t)}}\right\} \\
& \stackrel{\text { def }}{=} \sum_{s, t} f_{2}\left(\hat{\theta}_{s_{q(t)}}, y(t), \mathbf{x}(t)\right) w(s, t) .
\end{aligned}
$$

The function $w(s, t)$ introduced above is the total probability that the system is in state $s$ at time $t$, i.e. $w(s, t) \stackrel{\text { def }}{=} P_{s(t), y_{1}^{T}}\left(s, y_{1}^{T}\right)$.

To minimize $F_{2}(\hat{\theta})$ is the same as to find values of $\bar{y}_{s}$ and a that minimize

$$
\chi^{2}=\sum_{t}\left\{y(t) \sqrt{w(s, t)}-\left(\bar{y}_{s}+\mathbf{x}(t) \cdot \mathbf{a}_{s}\right) \sqrt{w(s, t)}\right\}^{2}
$$

which can be solved by using Singular Value Decomposition (SVD) [16], and $\sigma_{s}$ can be set by

$$
\sigma_{s}=\sqrt{\frac{1}{W(s)} \sum_{t} w(s, t)\left(y(t)-\hat{y}_{s}(t)\right)^{2}}
$$


where $W(s) \stackrel{\text { def }}{=} \sum_{t} w(s, t)$.

IV.2.4 The Forward Backward Algorithm

The maximization of $F_{1}(\hat{\theta})$ can be solved by the FBA which depends on the $\alpha$ s and $\beta$ s defined as follows:

$$
\begin{aligned}
& \alpha(s, t) \stackrel{\text { def }}{=} P_{y_{1}^{t}, s(t)}\left(y_{1}^{t}, s\right) \\
& \beta(s, t) \stackrel{\text { def }}{=} P_{y_{t+1}^{T} \mid s(t), y_{1}^{t}}\left(y_{t+1}^{T}, s, y_{1}^{t}\right)
\end{aligned}
$$

Thus

$$
w(s, t)=\alpha(s, t) \beta(s, t)
$$

If we define $w(i, j, t)=P_{s(t+1), s(t), y_{1}^{T}}\left(i, j, y_{1}^{T}\right)$, then

$$
w(i, j, t)=\alpha(j, t) P_{y \mid s, \mathbf{x}}(y(t+1), i, \mathbf{x}(t+1)) P_{s(2) \mid s(1)}(i, j) \beta(i, t+1)
$$

Here the transition probabilities are $P_{s(2) \mid s(1)}(i, j) \propto \sum_{t} w(i, j, t)$ subject to normalization. The $\alpha$ s and $\beta$ s can be evaluated by the recursion formulas as follows:

$$
\begin{gathered}
\alpha(s, t)=\sum_{j} \alpha\left(s_{j}, t-1\right) P_{s(2) \mid s(1)}\left(s, s_{j}\right) P_{y(1) \mid s(1), \mathbf{x}(1)}(y(t), s, \mathbf{x}(t)) \\
\beta(s, t)=\sum_{j} \beta\left(s_{j}, t+1\right) P_{s(2) \mid s(1)}\left(s_{j}, s\right) P_{y(1) \mid s(1), \mathbf{x}(1)}\left(y(t+1), s_{j}, \mathbf{x}(t+1)\right) .
\end{gathered}
$$

The initial conditions are set as follows:

- The initial state probabilities are

$$
P_{s(1), \hat{\theta}}(s) \propto w(s, 1)=\alpha(s, 1) \beta(s, 1)
$$

subject to normalization.

- $\alpha(s, 1)=P_{s(1)}(s) P_{y \mid s, \mathbf{x}}(y(1), s, \mathbf{x}(1))$. 
- $\beta(s, T)=1$.

At last the likelihood can be evaluated as

$$
P_{\theta}\left(y_{1}^{T}\right)=\sum_{s} \alpha(s, T)
$$

\section{IV.3 DETECTION OF CHAOTIC SIGNALS}

\section{IV.3.1 Detection Methods}

We have used the following three model building methods:

I. Vector Quantization (VQ) followed by the FBA.

1. Vector quantize. $\mathbf{R}^{D+1}$ is partitioned on the basis of observed vectors of the form $z(t)=y_{t-D}^{t}$. A variant of Lloyd algorithm [17] is used in which the metric inside each partition element is set proportional to the inverse covariance matrix of the observations in that partition element, and the determinants of all of the metric matrices are normalized to unity.

2. Fit transition probabilities. The state transition probabilities $P_{s(2) \mid s(1)}(i, j)$ are set to the relative frequencies with which sequential vectors $z(t+1), z(t)$ fall in partition elements $i, j$.

3. Fit outputs. For each partition element, the density $p(y(t) \mid \mathbf{x}(t))$ is fit on the basis of the $z(t) \mathrm{s}$ that fall in the element.

4. Train the model with FBA for 30 iterations which gave convergence tolerance roughly to 0.001 , i.e., $\left|\frac{\Delta P_{\theta}\left(y_{1}^{T}\right)}{P_{\theta}\left(y_{1}^{T}\right)}\right| \leq 0.001$. 
II. Noiseless training with model parameters adjusted for noise.

1. Begin with a model generated by Method $\mathbf{I}$ applied to a sample of noise free signal.

2. Change $\mathbf{a}_{s}$ and $\sigma_{s}$ in each state to values determined by the test signal to maximize the likelihood. The procedure is discussed as follows:

If the noise is from a 15 th order AR model, a good HFHMM means when $D \geq 15$ there should be only one discrete state no matter what the state transition probabilities could be, i.e., all $\bar{y}_{s}, \mathbf{a}_{s}$ and $\sigma_{s}$ are the same for all $s$, on the other hand when $D<15$ there will be some differences among them, but compared to the model trained from the signal, these differences will be so small that they can be ignored. In a later subsection we will show that this assumption is verified by the model performances.

As described before, $\mathbf{a}_{s}$ and $\sigma_{s}$ can be set using Equation 25 and 26, which is equivalent to solving the Normal (Yule-Walker) Equations with minor modifications

$$
\mathrm{r}_{s}=\mathbf{R}_{s} \mathbf{a}_{s}
$$

where

$$
\mathbf{R}_{s}=\left(\begin{array}{cccc}
r_{s}(0) & r_{s}(1) & \cdots & r_{s}(D-1) \\
r_{s}(1) & r_{s}(0) & \cdots & r_{s}(D-2) \\
\cdot & \cdot & \cdot & \cdot \\
\cdot & \cdot & \cdot & \cdot \\
\cdot & \cdot & \cdot & \cdot \\
r_{s}(D-1) & r_{s}(D-2) & \cdots & r_{s}(0)
\end{array}\right)
$$

Instead of $r(k)=\left\langle y^{*}(t) y(t-k)\right\rangle$, we use $r_{s}(k)=\left\langle w(s, t) y^{*}(t) y(t-k)\right\rangle$ where $w(s, t)$ comes from the FBA on noise free signal. Let $\mathbf{r}_{s}^{1}$ and $\mathrm{r}_{s}^{0}$ denote the 
ACF of the signal $y_{1}(t)$ and noise $y_{0}(t)$ at state $s$ respectively. The mixed signal $y^{\prime}(t)=\sqrt{\lambda} y_{1}(t)+\sqrt{1-\lambda} y_{0}(t)(0 \leq \lambda \leq 1)$ whose ACF at state $s$ is

$$
\mathbf{r}_{s}^{\prime}=\lambda \mathbf{r}_{s}^{1}+(1-\lambda) \mathbf{r}_{s}^{0}
$$

and

$$
\mathbf{R}_{s}^{\prime}=\lambda \mathbf{R}_{s}^{1}+(1-\lambda) \mathbf{R}_{s}^{0}
$$

From Equation 27 we get

$$
\mathbf{a}_{s}^{\prime}=\mathbf{R}_{s}^{\prime-1} \mathbf{r}_{s}^{\prime}
$$

and $\sigma_{s}^{\prime}$ is set as

$$
\sigma_{s}^{\prime}=\sqrt{r_{s}^{\prime}(0)-\sum_{i=1}^{D} a_{s}^{\prime}(i) r_{s}^{\prime}(i)}
$$

The other parameters such as $P(s(t+1) \mid s(t))$ and $\bar{y}_{s}$ remain the same as those in the model for a clean signal.

III. Noiseless training with higher order AR functions.

1. Begin with a model generated by Method I with lower order $L D$ AR functions.

2. Fill $a_{s}(i)$ with zeros where $L D<i \leq D$.

3. Use Method II step 2 to adjust $\mathrm{a}_{s}$ and $\sigma_{s}$.

Method I can be applied to a noise free signal, pure noise or a noisy signal. In the next section, when we refer to Method I, we mean the method applied to a noisy signal.

\section{IV.3.2 Detection Experiments}

In each of our detection experiments we used the following protocol for a fixed SNR. 
1. Use one of the methods described above and a given sample sequence to build a model of the combined signal and noise.

2. Train a HFHMM1 with the same number of parameters on the noise data.

3. Calculate likelihoods of each of the methods for several new sequences (usually 100 sequences with 4,000 points for each) of both noise alone and noisy signal.

4. On the basis of these likelihoods plot an experimental ROC.

Experimental ROCs by using different methods are plotted in 16 .

By using models with more free parameters, the detection performance can be improved. Figure 17 presents preliminary results of experiments with more complex models. As the number of model parameters is increased, large training data sets are required to prevent estimation error from degrading detector performance. The combination of a larger training set and a more complex model will achieve much better performance.

\section{IV.3.3 Discussions}

From the detection experiments it is clear that HFHMMs can detect differences which linear models miss. The reason is that though the PSDs of the signal and noise are similar in whole, at certain states different PSDs are estimated by the HFHMM. In order to show the different behaviors of signal and noise in HFHMM, we use plots as follow to illustrate.

First we divide the parameters of HFHMM into three classes:

1. State to state transition probabilities $P_{s(2) \mid s(1)}\left(s_{i}, s_{j}\right)$.

2. Output Gaussian distribution at state $s$ with constant part $\bar{y}_{s}$ and variance $\sigma_{s}^{2}$ 
3. AR coefficients $\mathbf{a}_{s}$ which determine the PSD of the AR model at state $s$.

We use the $P(s, t)=\sum_{j} P_{s(t) \mid s(t-1)}\left(s, s_{j}\right) P\left(s_{j}, t-1\right)$ to describe the characteristic of parameters of class 1 in Figure 18, where

$$
P(s, 1)=P_{s(1), \theta}(s) \propto w(s, 1)
$$

subject to normalization. Gaussian distribution $N\left(\bar{y}_{s}, \sigma_{s}^{2}\right)$ at state $s$ are present in Figure 19. We use PSDs of the AR models with $\mathbf{a}_{s}$ to represent the characteristics of AR coefficients in different states which are shown in Figure 20. All the plots are from models with 5 discrete states and third order AR functions, i.e. $\operatorname{HFHMM1}(3,5)$.

As described before for the noise which was generated from a 15 th order AR model, model parameters in each state $s$ should be the same. The plots verified the expectation. However, for the signal, we can see parameters vary with different states. From the point of the parameters, we can say HFHMMs are better than linear models in this detection problem. 


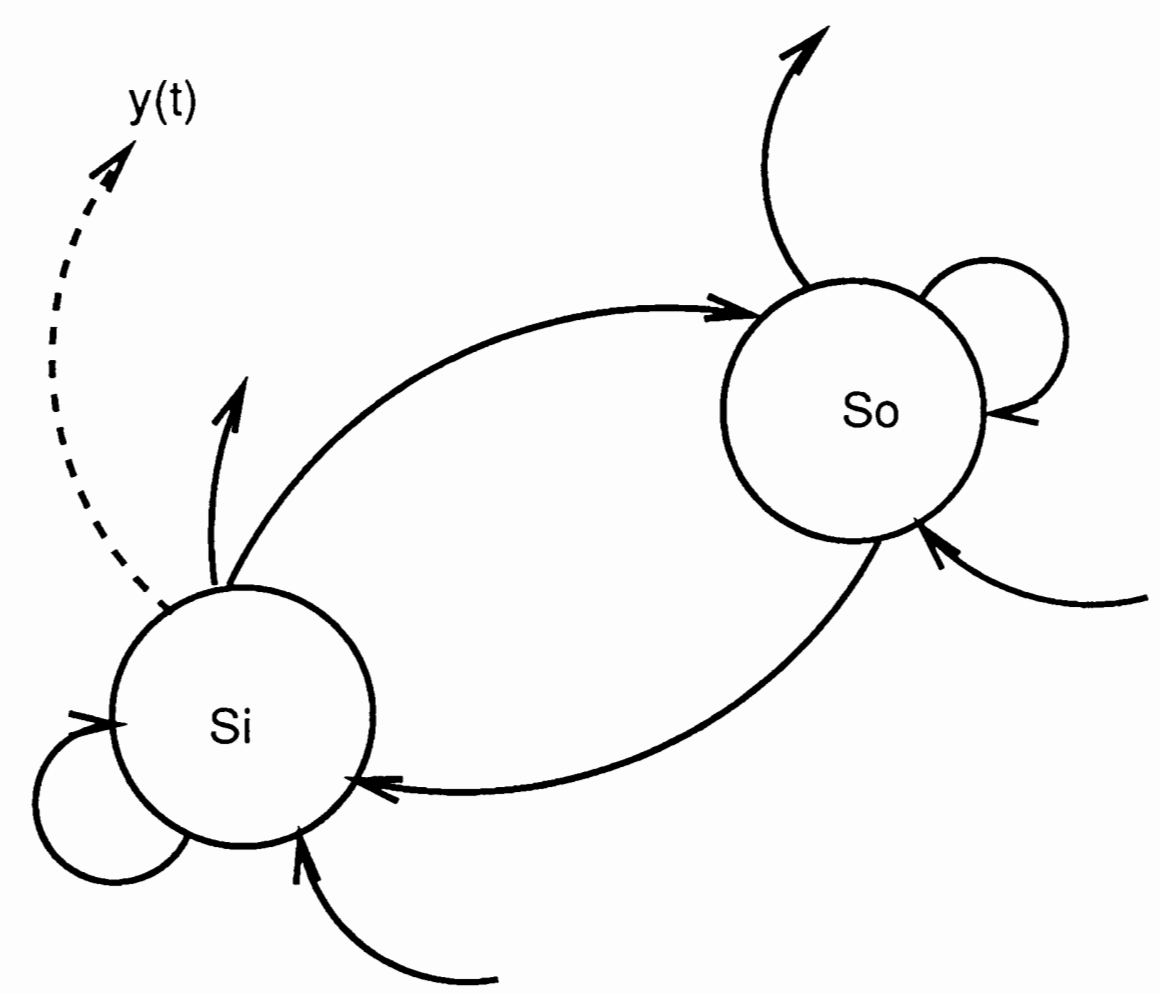

Figure 15. Components of a HMM, $s_{i}$ and $s_{0}$ denote different hidden states, solid arrows denote the transition probabilities between these states, $y(t)$ denotes the scalar output which depends on the current states and previous samples. 

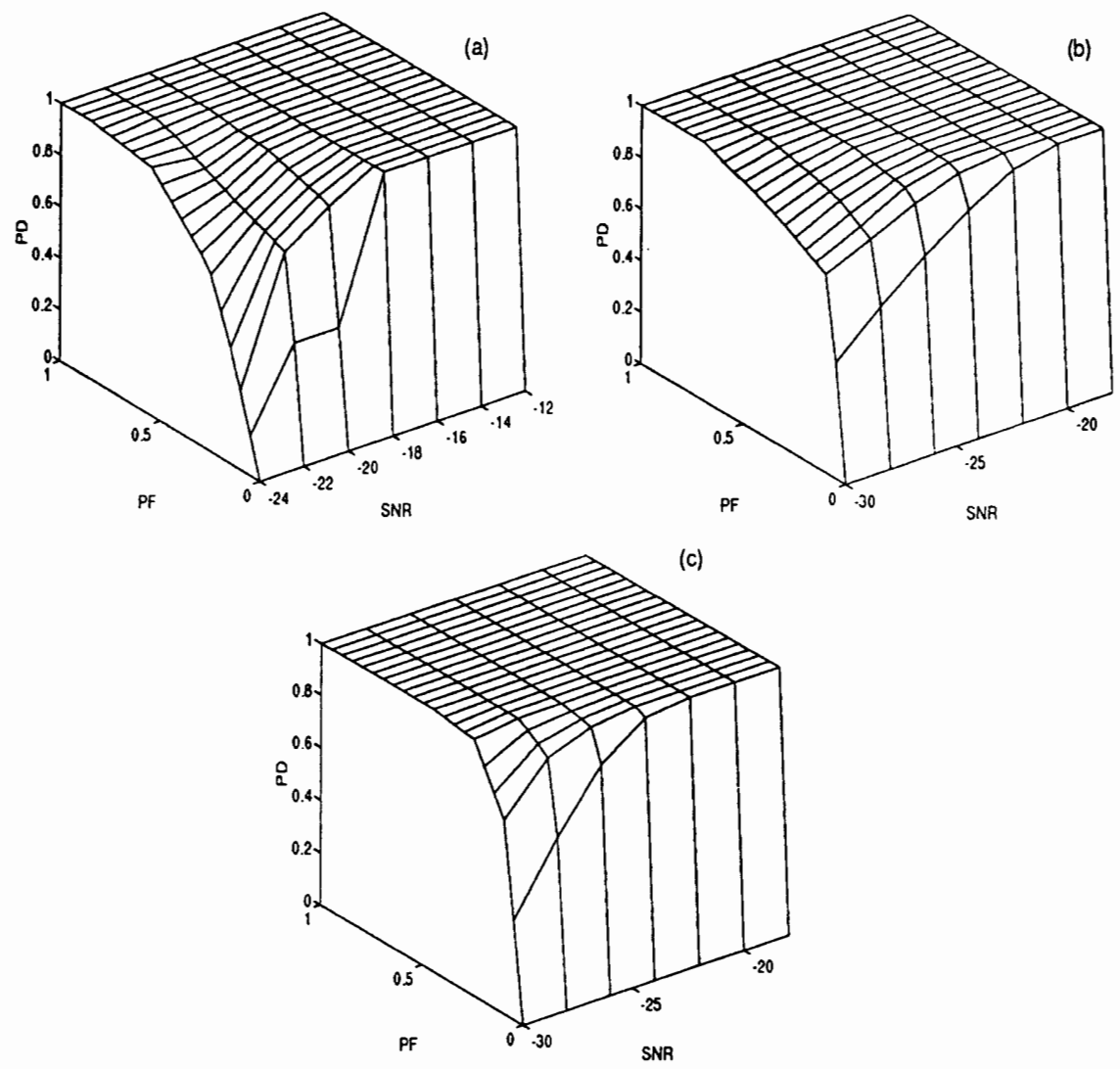

Figure 16. Experimental ROCs for detecting the noisy Duffing signal in 15th order AR noise using different methods. Training data length is 20,000 , testing data length is 4,000 . (a) HFHMM1 $(2,5)$ trained by using the VQ followed by the FBA, (b) HFHMM1 $(2,5)$ obtained by using noiseless training with model parameters adjusted for noise, (c) $\operatorname{HFHMM1}(15,5)$ based on $\operatorname{HFHMM1}(2,5)$ by using noiseless training with higher order AR functions. 


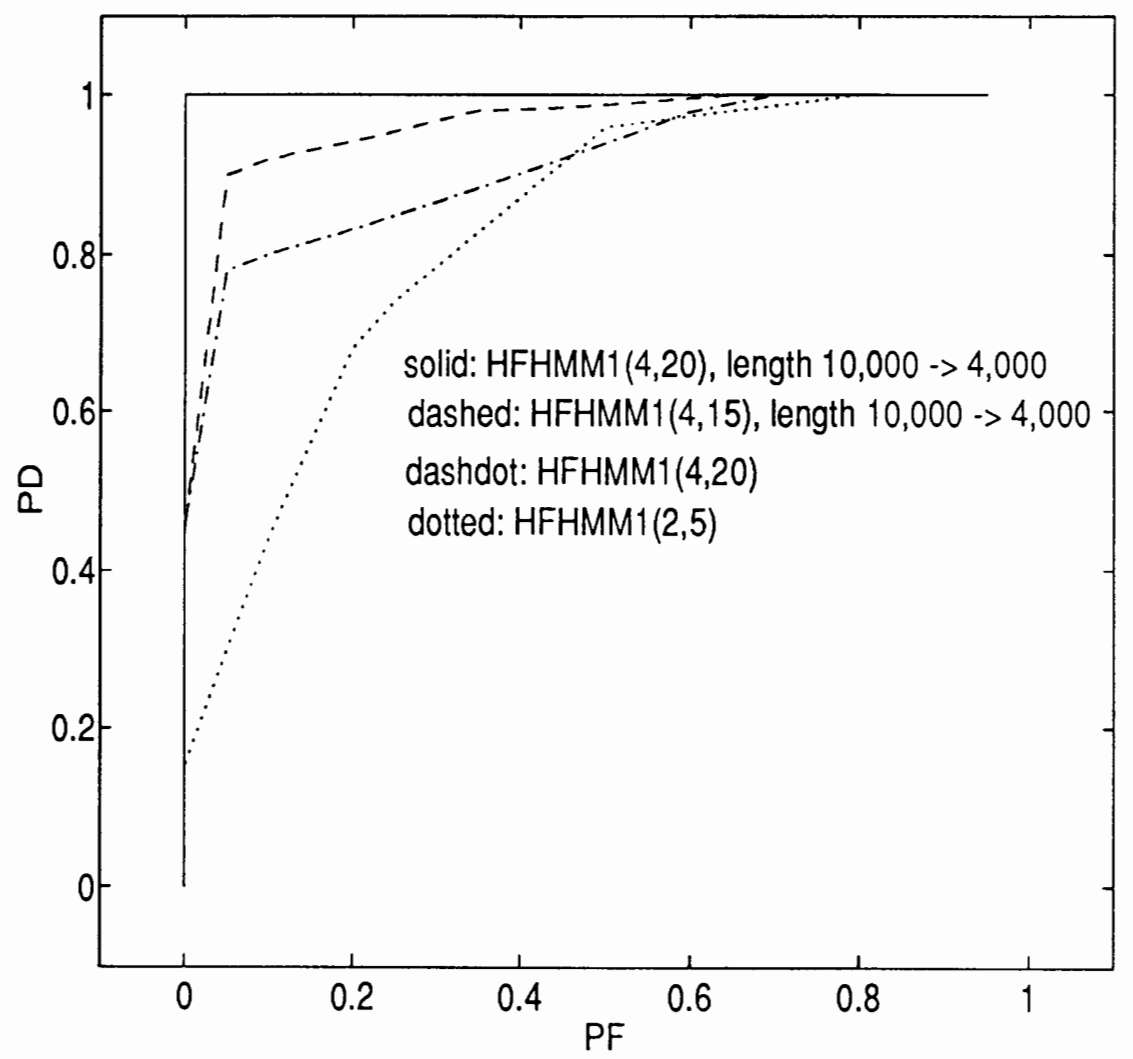

Figure 17. Different experimental ROCs using Method I at SNR of $-24 \mathrm{db}$, "length $T->M$ " denotes a model fit on length $T$ sequences and tested on length $M$ sequences, otherwise experiments are trained and tested on 4,000 samples. 

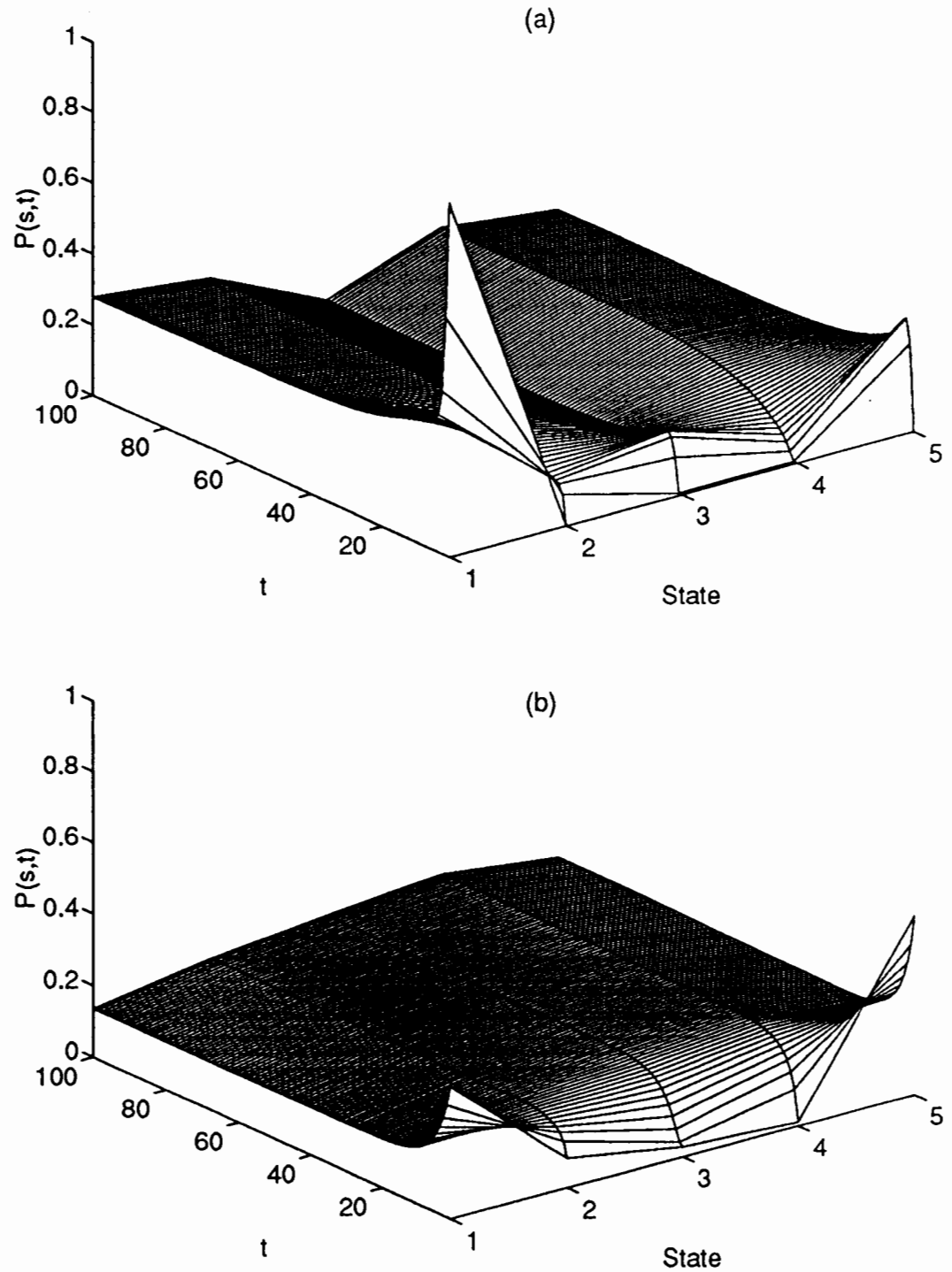

Figure 18. $P(s, t)$ obtained from $\operatorname{HFHMM} 1(3,5)$, (a) the Duffing signal, (b) the 15th order AR noise. 

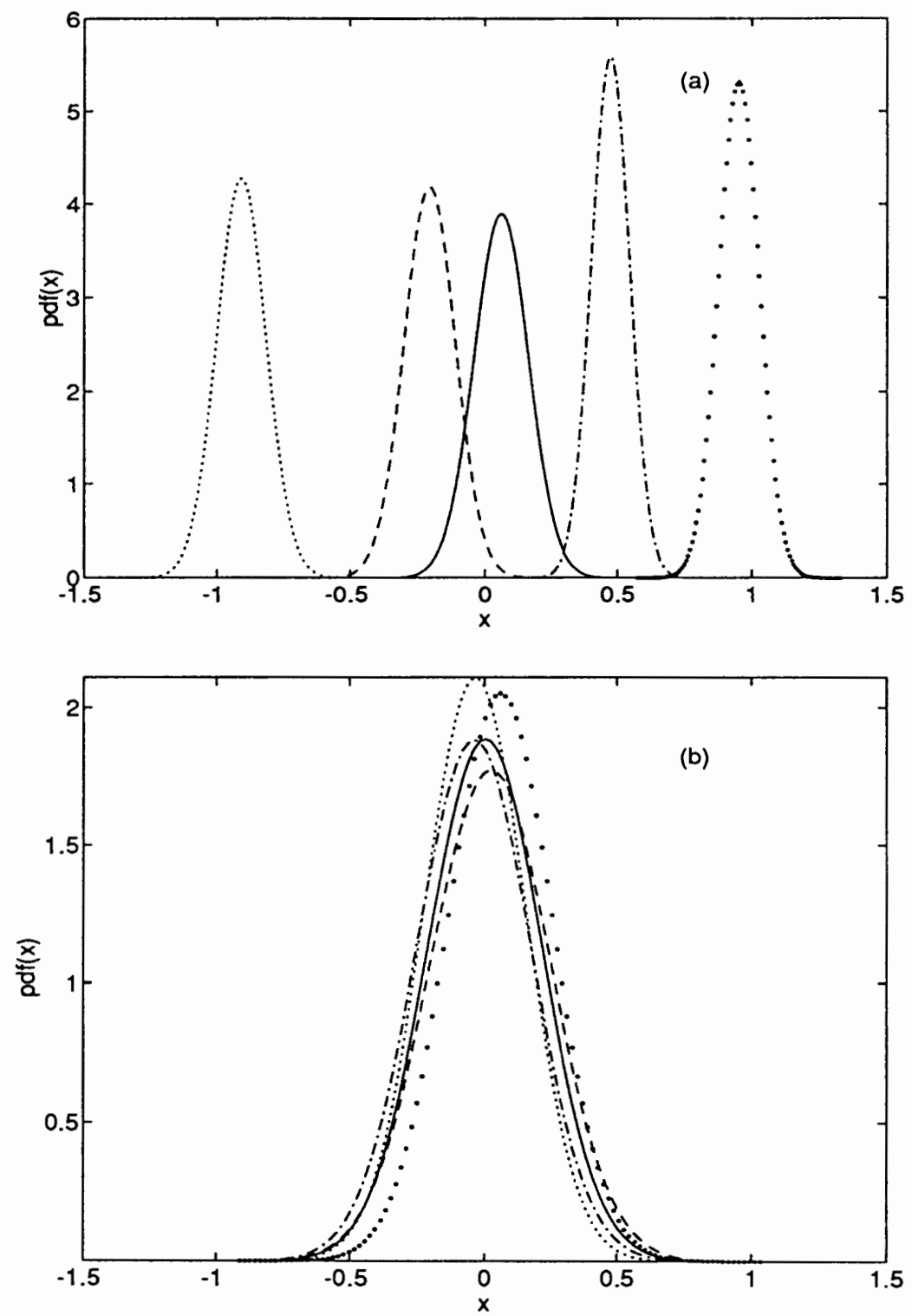

Figure 19. Output distribution $N\left(\bar{y}_{s}, \sigma_{s}^{2}\right)$ at state $s$, different line types denote different hidden states, (a) the Duffing signal, (b) the 15 th order AR noise. 

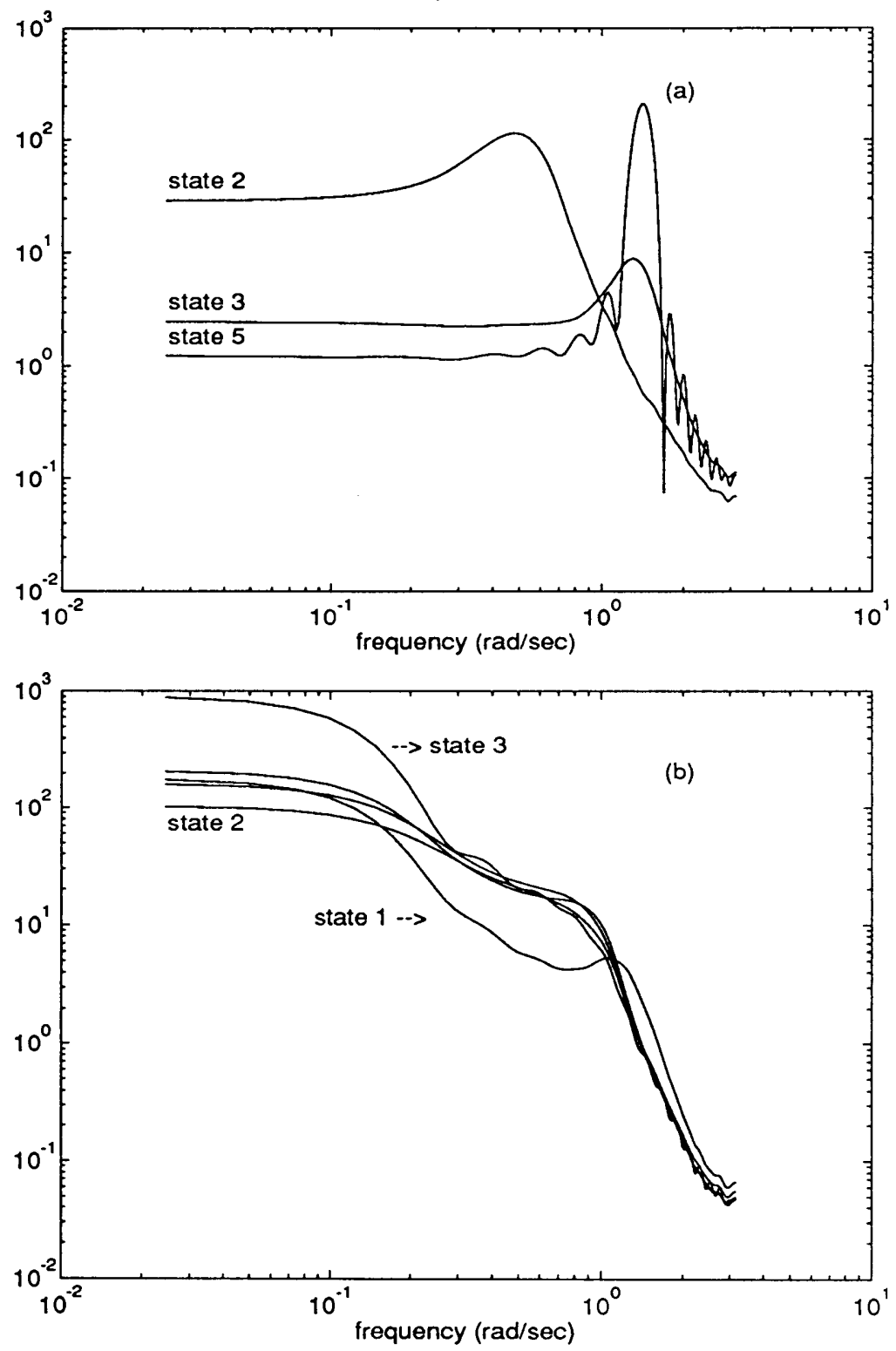

Figure 20. PSDs of AR models generated from $\mathbf{a}_{s}$, (a) the Duffing signal (state 1 and 4 are unplotted because of their unstability), (b) the 15th order AR noise. 


\section{CHAPTER V}

\section{HIDDEN FILTER HIDDEN MARKOV MODEL 2}

\section{V.1 INTRODUCTION}

After having built HFHMM1, the next natural step in the study was to improve it for better detection performance. An HMM consists of two parts: a hidden Markov chain and the output distribution. They will be examined individually to find out what can be done with respect to these two aspects. In [2] Fraser and Dimitriadis introduced Mixed State Hidden Markov Models (MSHMM) in which the sequence $(s(t), \mathbf{x}(t))$ constitutes a Markov chain instead of only $s(t)$ in HFHMM1. On the other hand, HFHMM2 deals with $P_{y(t) \mid s(t), \mathbf{v}(t)}$, the conditional distribution of the observation $y(t)$ given the discrete state $s$ and the context vector $\mathbf{v}(t)$, as a Gaussian with mean linearly depending on the context, $\hat{y}_{s}(t)=\bar{y}_{s}+\mathbf{a}_{s} \cdot \mathbf{v}(t)$. In HFHMM1 the context was simply a history vector $\mathrm{x}(t)=y_{t-D}^{t-1}$, but this representation is not always adequate. Sometimes observations over an interval $H D$ characteristic of the observation are required [11]. If we use the history vector $\mathbf{x}(t)=y_{t-H D}^{t-1}$, the number of free parameters $\left((H D+1) N_{s}\right.$, one added to include the constant $\left.\bar{y}_{s}\right)$ might be very large. One risks encountering the "overfit" problem, i.e., if the number of parameters is the same as or more than the length of the training sequence, the model will be badly fit. Compared to the number of training data, the fewer the parameters, the better the model was fit. So Fraser suggested finding a global map $\psi: \mathbf{R}^{H D} \rightarrow \mathbf{R}^{D}$ with $\mathbf{B x}(t)=\psi\left(y_{t-H D}^{t-1}\right)$ that provides a low dimensional context 
containing information from all important time scales. In the communities doing experiments with physical and numerical chaotic systems, such a map is called a reconstruction [18]. So in HFHMM2 a context vector $\mathbf{B x}(t)$ takes the place of the former history vector $\mathbf{x}(t)$. For tracing the same length of $H D$ of previous outputs, less free parameters $\left(H D \times D+(D+1) N_{s}\right)$ are required for large $N_{s}$ in HFHMM2. Thus, the Conjugate Gradient Method is used as a searching algorithm instead of simply adopting SVD in linear fitting.

\section{V.2 MODELING}

The model structure of HFHMM2 is similar to that of HFHMM1, except that the context vector is changed to $\mathbf{B x}(t)$ instead of the former $\mathbf{x}(t)$. In each iteration of FBA used to build HFHMM1, we must adjust the parameters to minimize

$$
F_{2}(\hat{\theta})=\sum_{s, t} w(s, t)\left\{\log \sigma_{s}+\frac{\left(y(t)-\bar{y}_{s}-\mathbf{a}_{s} \cdot \mathbf{B} \mathbf{x}(t)\right)^{2}}{2 \sigma_{s}^{2}}\right\},
$$

where $w(s, t)$ is the probability that the system is in state $s$ at time $t$.

It is the same as minimizing

$$
F(\hat{\theta})=\sum_{s}\left\|\mathbf{L}_{s}\right\|^{2}
$$

where $\mathbf{L}_{s}$ is 


$$
\begin{aligned}
& \left(\begin{array}{c}
y(1) \sqrt{w(s, 1)} \\
\cdot \\
\cdot \\
\cdot \\
\cdot \sqrt{w(s, t)} \\
\cdot \\
\cdot \\
\cdot \\
y(T) \sqrt{w(s, T)}
\end{array}\right)-\left(\begin{array}{cccc}
y(1-1) \sqrt{w(s, 1)} & \cdots & y(1-H D) \sqrt{w(s, 1)} & \sqrt{w(s, 1)} \\
\cdot & \cdot & \cdot & \cdot \\
\cdot \cdot(t-1) \sqrt{w(s, t)} & \cdots & y(t-H D) \sqrt{w(s, t)} & \sqrt{w(s, t)} \\
\cdot & \cdot & \cdot \\
\cdot & \cdot & \cdot \\
y(T-1) \sqrt{w(s, T)} & \cdots & y(T-H D) \sqrt{w(s, T)} & \sqrt{w(s, T)}
\end{array}\right) \\
& \times\left(\begin{array}{cccc}
b_{1,1} & \cdots & b_{1, D} & 0 \\
\cdot & & \cdot & \cdot \\
\cdot & & \cdot & \cdot \\
\cdot & & \cdot & \cdot \\
b_{H D, 1} & \cdots & b_{H D, D} & 0 \\
0 & \cdots & 0 & 1
\end{array}\right)\left(\begin{array}{c}
a_{s, 1} \\
\cdot \\
\cdot \\
\cdot \\
a_{s, D} \\
\bar{y}_{s}
\end{array}\right)
\end{aligned}
$$

And setting

$$
\sigma_{s}=\sqrt{\frac{1}{W(s)} \sum_{t} w(s, t)\left(y(t)-\hat{y}_{s}(t)\right)^{2}}
$$

with $W(s) \stackrel{\text { def }}{=} \sum_{t} w(s, t)$ and $\hat{y}_{s}(t)=\bar{y}_{s}+\mathbf{a}_{s} \cdot \mathbf{B} \mathbf{x}(t)$.

The key point is we need to minimize the $F(\hat{\theta})$ over $\mathbf{B}$ and all of the $\mathbf{a}_{s} \mathrm{~s}$, $\bar{y}_{s}$ s. From the above equation it is obvious that the solution is not unique. For example, if $\mathbf{B}, \mathbf{a}_{s}$ and $\bar{y}_{s}$ is one of the solutions, $\lambda \mathbf{B}, \frac{1}{\lambda} \mathbf{a}_{s}$ and $\frac{1}{\lambda} \bar{y}_{s}$ with any constant $\lambda \neq 0$ is another solution. Finally we decided to search by using Conjugate Gradient Methods (CGM) [16] followed by linear fitting of SVD.

The CGM is to minimize the quadratic form

$$
f(\mathbf{x}) \approx c-\mathbf{b} \cdot \mathbf{x}+\frac{1}{2} \mathbf{x} \cdot \mathbf{A} \cdot \mathbf{x}
$$

where $c=f(P), b_{i}=\left.\frac{\partial f}{\partial x_{i}}\right|_{P}$ and $A_{i, j}=\left.\frac{\partial^{2} f}{\partial x_{i} \partial x_{j}}\right|_{P}$. The algorithm can be described briefly as follows: 
1. Start with an initial point $P_{0}$, and let the starting conjugate direction $h_{0}=g_{0}$ be an arbitrary vector.

2. Move $P_{i-1}$ to $P_{i}$ along the conjugate direction of $h_{i}$, where $h_{i+1}=g_{i+1}+v_{i} h_{i}$ and $v_{i}=\frac{g_{i+1} \cdot g_{i+1}}{g_{i} \cdot g_{i}}$. Set $g_{i+1}=-\nabla f\left(P_{i+1}\right)$.

3. If $\left|f\left(P_{i}\right)-f\left(P_{i-1}\right)\right| \geq F T O L\left(\left|f\left(P_{i}\right)\right|+\left|f\left(P_{i-1}\right)\right|\right)$ (FTOL is converge intolerance), then $i=i+1$, go to 2 . Otherwise stop.

In Conjugate Gradient Methods the value of the function and its first derivative are needed

$$
\begin{aligned}
\frac{\partial F_{2}}{\partial b_{i^{\prime}, j^{\prime}}} & =2 \sum_{s} a_{s}\left(j^{\prime}\right)\left\{\sum_{i=1}^{H D} \Phi_{s}\left(i, i^{\prime}\right) \sum_{j=1}^{D} b_{i, j} a_{s}(j)+\bar{y}_{s} Y_{s}\left(i^{\prime}\right)-\Phi_{s}\left(0, i^{\prime}\right)\right\} \\
\frac{\partial F_{2}}{\partial a_{s^{\prime}}\left(j^{\prime}\right)} & =2 \sum_{k=1}^{H D} b_{k, j^{\prime}}\left\{\sum_{i=1}^{H D} \Phi_{s}(i, k) \sum_{j=1}^{D} b_{i, j} a_{s^{\prime}}(j)+\bar{y}_{s^{\prime}} Y_{s^{\prime}}(k)-\Phi_{s^{\prime}}(0, k)\right\} \\
\frac{\partial F_{2}}{\partial \bar{y}_{s^{\prime}}} & =2\left\{\sum_{i=1}^{H D} Y_{s^{\prime}}(i) \sum_{j=1}^{D} b_{i, j} a_{s^{\prime}}(j)-\bar{y}_{s^{\prime}} W_{s^{\prime}}-Y_{s^{\prime}}(0)\right\}
\end{aligned}
$$

where

$$
\begin{aligned}
W_{s} & =\sum_{t} w(s, t) \\
Y_{s}(i) & =\sum_{t} w(s, t) y(t-i) \\
\Phi_{s}(i, j) & =\sum_{t} w(s, t) y(t-i) y(t-k)
\end{aligned}
$$

In this method we have encountered the local minimum problem which makes the selection of the initial point crucial to the searching. After doing the test, we think it is a problem but not serious enough to force us to discard the method. Table I and Table II list the results from different starting points, one is a delay coordinate, the other is a randomly selected one. In the tables described above, 
iterations denotes the iterations the CGM need to find the final position with $1 \times 10^{-4}$ of converge intolerance and 5 hidden states. We can see for a small number of parameters, they almost fall into similar minimum value, but for a large number of parameters an optimal starting point is more important.

On the other hand, in order to verify how good the CGM described above actually is, we use SVD as a comparison which minimizes the term on the right side of equation 28, i.e., to omit the $\mathbf{B}$ matrix or make it a $H D \times H D$ identity matrix, and $\mathbf{a}_{s}=\left[a_{s, 1}, a_{s, 2}, \ldots a_{s, H D}\right]^{T}$. Then we use SVD to calculate $\mathbf{a}_{s}$ here. The $w(s, t) \mathrm{s}$ are generated from FBA in $\operatorname{HFHMM1}(2,5)$ first. The comparison among different methods is shown in Table III, where $N_{M}$ denotes the number of parameters in Method $M(M$ could be SVD or CGM when $D=2$ or $D=3), F_{M}$ represents the final value of $F(\hat{\theta})$ in Method $M$. Though we know that the SVD method will be the best, i.e., $F_{S V D}$ is less than $F_{D=2}$ or $F_{D=3}$ under the same dimension $H D$. It turned out that the differences among SVD and CGMs are small. Compared to the number of parameters saved, we believe CGM is worth trying.

\section{V.3 DETECTION OF CHAOTIC SIGNALS}

\section{V.3.1 Detection Methods}

We have used four model building methods:

I. VQ followed by FBA.

Step 1, 2 and 3 are similar to that of Method $\mathbf{I}$ in Chapter IV.

4. Train the model with the FBA for 30 iterations. For each iteration use CGM to search the optimal parameters.

II. Noiseless training with model parameters adjusted for noise. 
1. Begin with a model generated by Method I applied to samples of the signal alone.

2. For given $\mathbf{B}$ from step 1 , adjust $\mathbf{a}_{s}$ and $\sigma_{s}$ in each state to values determined by the test signal to maximize the likelihood. The procedure is similar to what we have described in Method II of Chapter IV. The only difference is due to the introduction of the $\mathbf{B}$ matrix, thus we proceed as follows:

$$
\mathbf{h}_{s}=\mathbf{R}_{s} \cdot \mathbf{B}^{T} \cdot \mathbf{a}_{s}=\Psi_{s} \mathbf{a}_{s}
$$

where

$$
\mathbf{R}_{s}=\left(\begin{array}{cccc}
r_{s}(0) & r_{s}(1) & \cdots & r_{s}(H D-1) \\
r_{s}(1) & r_{s}(0) & \cdots & r_{s}(H D-2) \\
\cdot & \cdot & \cdot & \cdot \\
\cdot & \cdot & \cdot & \cdot \\
\cdot & \cdot & \cdot & \cdot \\
r_{s}(H D-1) & r_{s}(H D-2) & \cdots & r_{s}(0)
\end{array}\right)
$$

and $h_{s}=\left[r_{s}(1), r_{s}(2), \ldots, r_{s}(H D)\right]^{T}$.

$$
\boldsymbol{\Psi}_{s}=\mathbf{R}_{s} \cdot \mathbf{B}^{T}=\left(\begin{array}{cccc}
\rho_{s}(1,1) & \rho_{s}(1,2) & \cdots & \rho_{s}(1, D) \\
\rho_{s}(2,1) & \rho_{s}(2,2) & \cdots & \rho_{s}(2, D) \\
\cdot & \cdot & \cdot & \cdot \\
\cdot & \cdot & \cdot & \cdot \\
\cdot & \cdot & \cdot & \cdot \\
\rho_{s}(D, 1) & \rho_{s}(D, 2) & \cdots & \rho_{s}(D, D)
\end{array}\right)
$$

with

$$
\rho_{s}(k, i)=\sum_{j=1}^{H D} r_{s}(k-j) b_{j, i} .
$$

Therefore from Equation 31, we get equation

$$
\mathrm{r}_{s}=\Psi_{s} \mathrm{a}_{s}
$$

with $\mathbf{r}_{s}=\left[r_{s}(1), r_{s}(2), \ldots, r_{s}(D)\right]^{T}$ 
For noisy signal

$$
\begin{aligned}
\mathrm{a}_{s}^{\prime} & =\Psi_{s}^{\prime-1} \mathrm{r}_{s}^{\prime} \\
\sigma_{s}^{\prime} & =\sqrt{r_{s}^{\prime}(0)-\sum_{i=1}^{D} a_{s}^{\prime}(i) \rho_{s}^{\prime}(0, i)}
\end{aligned}
$$

with

$$
\begin{aligned}
\rho_{s}^{\prime}(k, i) & =\sum_{j=1}^{H D} r_{s}^{\prime}(k-j) b_{j, i} \\
& =\sum_{j=1}^{H D}\left\{\lambda r_{s}^{1}(k-j)+(1-\lambda) r_{s}^{0}(k-j)\right\} b_{j, i}
\end{aligned}
$$

The other parameters such as $P(s(t) \mid s(t-1))$ and $\bar{y}_{s}$ remain the same.

III. Retraining on noisy data.

1. Begin with a model generated by Method II.

2. Train the model with FBA and CGM for several iterations.

IV. Vector quantization on given B followed by FBA on noisy data.

1. Vector quantize on the basis of the $\mathbf{B}$ from Method II step $1 . \mathbf{R}^{D+1}$ is partitioned on the basis of the form $z(t)=[y(t), v(t, 1), v(t, 2), \ldots, v(t, D)]^{T}$, where $v(t, i)=\sum_{j=1}^{H D} y(t-j) b_{j, i}, i=1,2, \ldots, D$. Then a variant of the Lloyd algorithm is used in which the metric inside each partition element is set proportional to the inverse covariance matrix of the observations in that partition element, and the determinates of all of the metric matrices are normalized to unity.

2. The remaining steps are as same as described in Method I.

\section{V.3.2 Experiment Results}

We use the same protocol described in Chapter IV in each of our detection experiments for a fixed SNR. 
In Figure 21 to 25, HFHMM2 $\left(H D \rightarrow D, N_{s}\right)$ denotes Hidden Filter Hidden Markov Model 2 with $H D$ as the higher order, $D$ as the lower order of AR functions and $N_{s}$ discrete states. Figure 21 presents experimental ROCs in HFHMM2(10 $\rightarrow 3$, 10) compared to HFHMM1 $(6,10)$ which has the same number of parameters for the same range of SNR. In Figure 21.b the models were built by VQ followed by FBA on noisy data. Obviously the detection performance of HFHMM2 is better.

Figure 22 is an experimental ROC obtained by using Method I with SNR from $-18 \mathrm{db}$ to $-30 \mathrm{db}$. Experimental ROCs from Method II, III and IV are plotted in Figure 23, 24 and 25 respectively.

The results using Method II are not as good as we expected. The reason for the poorer performance is that in deriving Equation 32 from Equation 31, i.e., from

$$
\mathbf{h}_{s}=\mathbf{R}_{s} \cdot \mathbf{B}^{T} \cdot \mathbf{a}_{s}
$$

to

$$
\mathrm{r}_{s}=\mathbf{\Psi}_{s} \mathrm{a}_{s}
$$

the number of equation sets is reduced from $H D$ to $D$, thus we lose some information which causes the misadjustment of model parameters. However, we can see that the detection performances in all HFHMM2s are better than that of HFHMM1 with same number of parameters. 
TABLE I

TEST RESULT TRAINING ON 4,000 SAMPLES WITH 5 HIDDEN STATES BY STARTING FROM DELAY COORDINATES

\begin{tabular}{|c|c|c|c|c|c|}
\hline \hline$D$ & $H D$ & time(mins) & iterations & original $F(\hat{\theta})$ & final $F(\hat{\theta})$ \\
\hline \hline 2 & 3 & 6 & 26 & 409.386017 & 81.876968 \\
2 & 4 & 8 & 28 & 409.390778 & 75.486465 \\
2 & 5 & 12 & 35 & 409.419495 & 74.998726 \\
2 & 6 & 17 & 44 & 409.463104 & 71.733971 \\
2 & 7 & 21 & 48 & 409.588501 & 69.833961 \\
2 & 8 & 41 & 77 & 409.578308 & 64.528053 \\
\hline 3 & 3 & 11 & 40 & 3445.578125 & 82.139679 \\
3 & 4 & 9 & 27 & 3444.349121 & 75.559464 \\
3 & 5 & 13 & 29 & 3443.077148 & 76.730659 \\
3 & 6 & 16 & 31 & 3443.099854 & 75.480049 \\
3 & 7 & 21 & 34 & 3443.343506 & 69.108902 \\
3 & 8 & 27 & 38 & 3443.500732 & 65.854065 \\
\hline \hline
\end{tabular}

TABLE II

TEST RESULT TRAINING ON 4,000 SAMPLES WITH 5 HIDDEN STATES BY STARTING FROM RANDOM COORDINATES

\begin{tabular}{|c|c|c|c|c|c|}
\hline \hline$D$ & $H D$ & time(mins) & iterations & original $F(\hat{\theta})$ & final $F(\hat{\theta})$ \\
\hline \hline 2 & 3 & 7 & 30 & 74512.835938 & 89.071007 \\
\hline 3 & 3 & 11 & 38 & 226962.796875 & 85.729912 \\
3 & 4 & 30 & 82 & 810022.625000 & 77.378387 \\
3 & 5 & 23 & 49 & 884637.312500 & 81.546204 \\
3 & 6 & 57 & 100 & 683397.750000 & 226.059021 \\
\hline \hline
\end{tabular}


TABLE III

COMPARISON BETWEEN SVD AND CGM TRAINING ON 4,000 SAMPLES WITH 5 HIDDEN STATES

\begin{tabular}{|c|c|c|c|c|c|c|}
\hline \hline$H D$ & $N_{S V D}$ & $N_{D=2}$ & $N_{D=3}$ & $F_{S V D}$ & $F_{D=2}$ & $F_{D=3}$ \\
\hline \hline 3 & 20 & 21 & 29 & 78.993011 & 81.876968 & 82.139679 \\
4 & 25 & 23 & 32 & 73.794121 & 75.486465 & 75.559464 \\
5 & 30 & 25 & 35 & 73.075951 & 74.998726 & 76.730659 \\
6 & 35 & 27 & 38 & 68.612015 & 71.733971 & 75.480049 \\
7 & 40 & 29 & 41 & 63.756989 & 69.833961 & 69.108902 \\
8 & 45 & 31 & 44 & 59.801315 & 64.528053 & 65.854065 \\
9 & 50 & 33 & 47 & 59.722061 & 63.880306 & 65.430412 \\
10 & 55 & 35 & 50 & 59.644306 & 64.980164 & 65.492989 \\
\hline
\end{tabular}



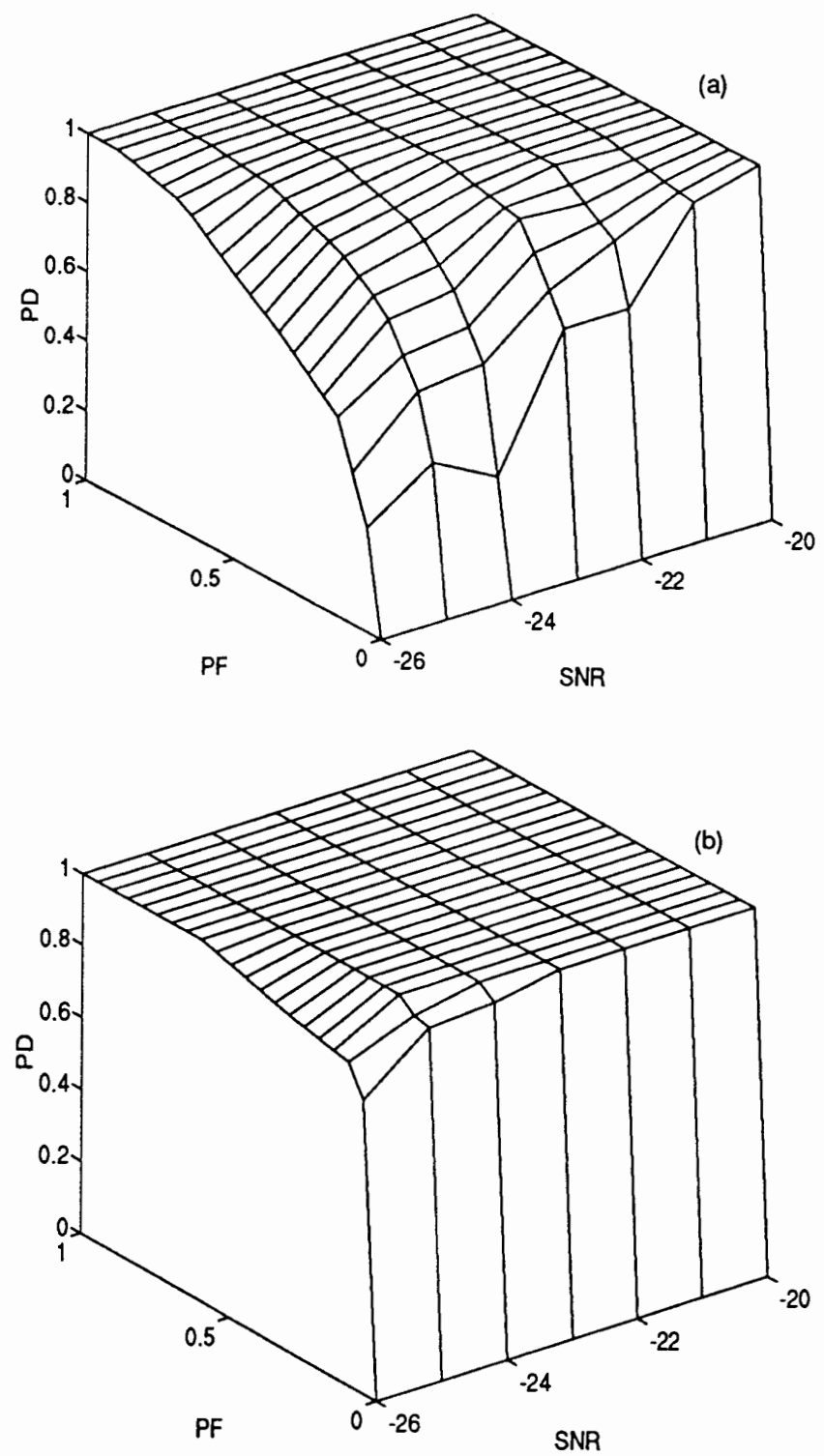

Figure 21. Experimental ROCs for detecting the noisy Duffing signal in 15th order AR noise using Method I and model: (a) HFHMM1(6,10), (b) HFHMM2 $(10 \rightarrow 3,10)$. 


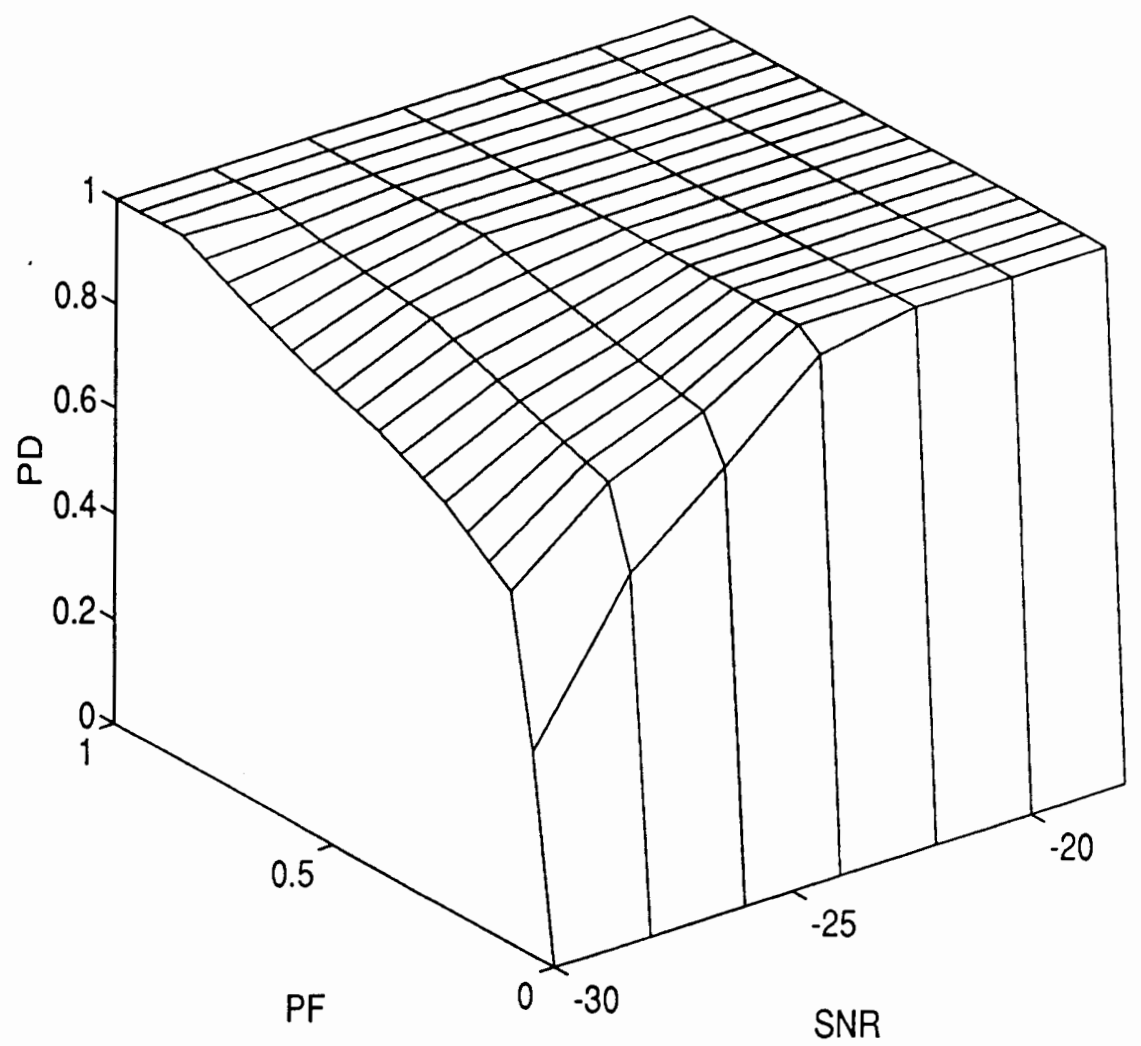

Figure 22. Experimental ROCs for detecting the noisy Duffing signal in 15th order AR noise using Method $\mathbf{I}$ and HFHMM2 $(10 \rightarrow 3,10)$. 


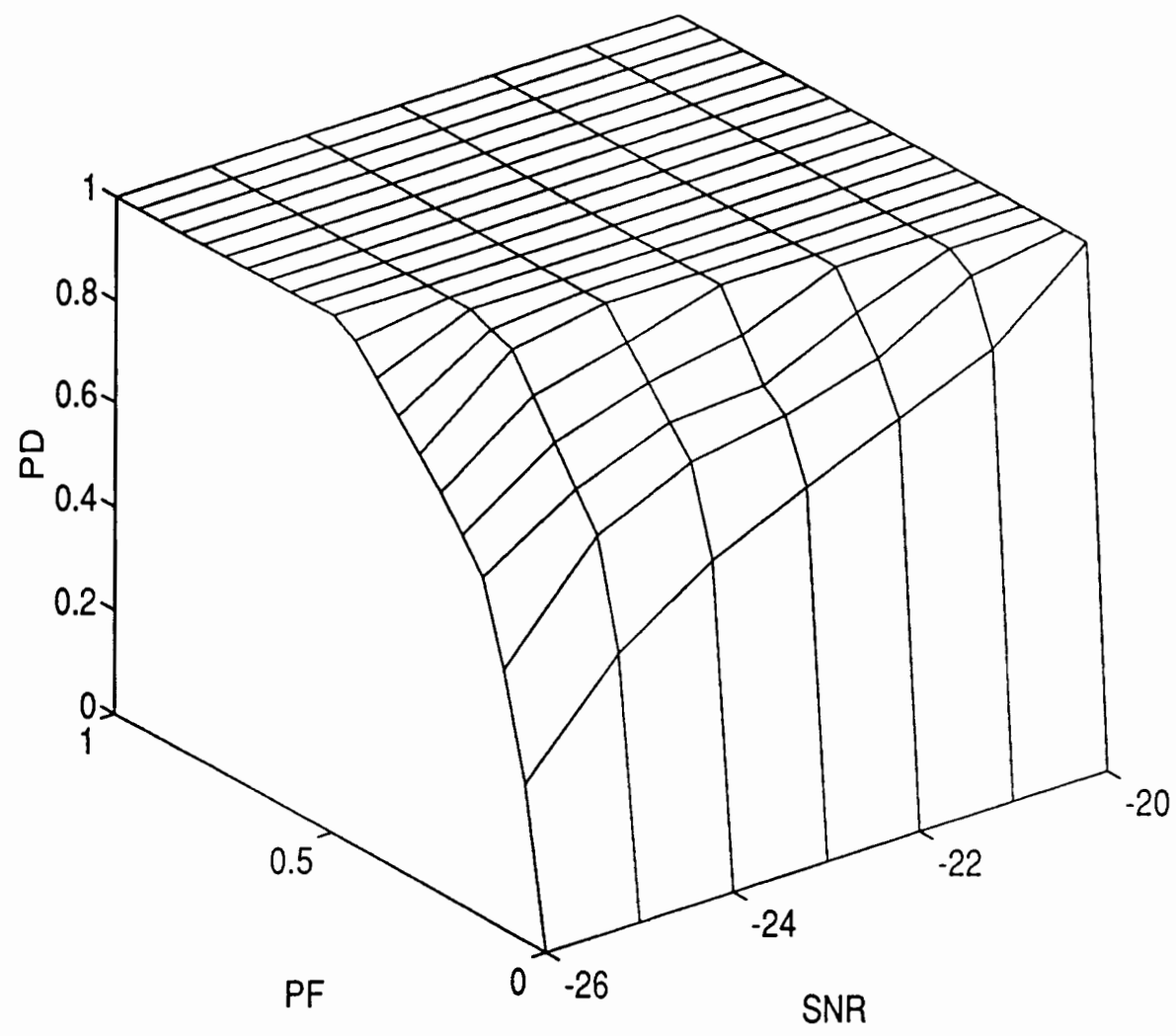

Figure 23. Experimental ROCs for detecting the noisy Duffing signal in 15 th order AR noise using Method II and HFHMM2 $(10 \rightarrow 3,10)$. 


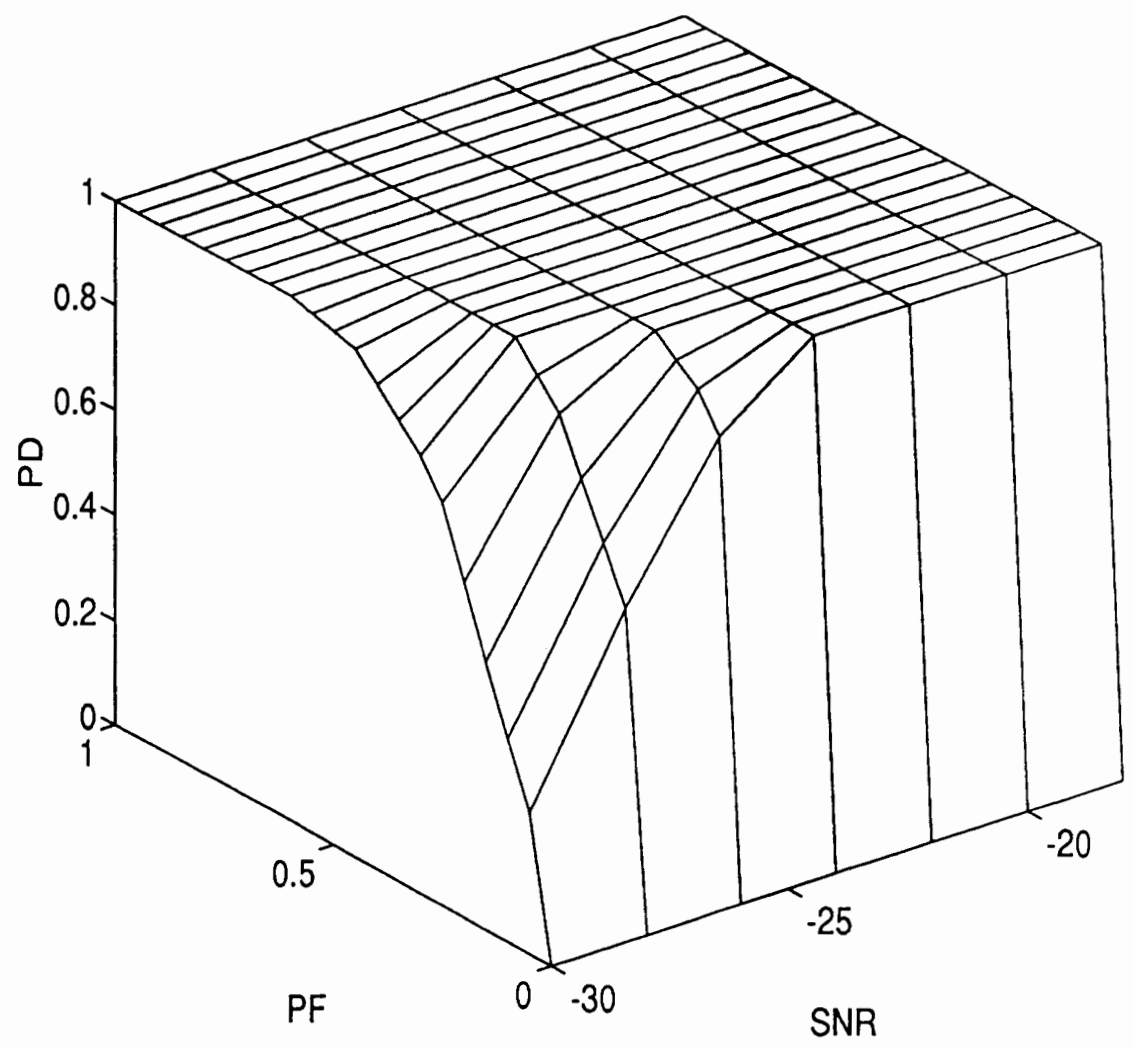

Figure 24. Experimental ROCs for detecting the noisy Duffing signal in 15th order AR noise using Method III and HFHMM2 $(10 \rightarrow 3,10)$. 


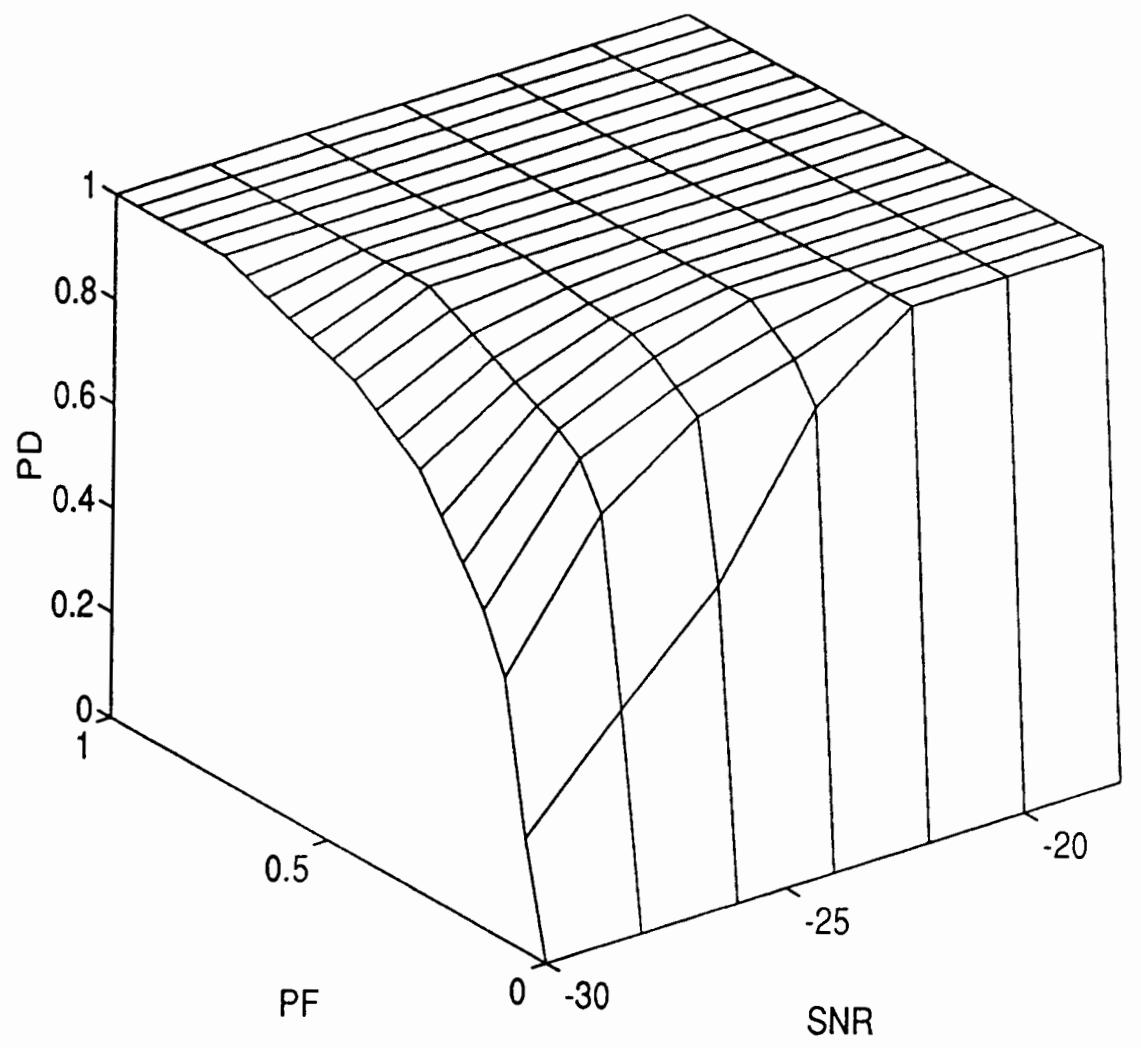

Figure 25. Experimental ROCs for detecting the noisy Duffing signal in 15th order AR noise using Method IV and HFHMM2 $(10 \rightarrow 3,10)$. 


\section{CHAPTER VI}

\section{CONCLUSION}

It was observed that strange or chaotic attractors are ubiquitous in nature as reported in scientific literature and they are theoretically generic in the space of differential equations. In this thesis, we have exploited these features of given signals and developed several nonlinear models for signal detection. HFHMM2 had the best overall detection performance among all the models studied. HFHMM1 was superior to the simple HMs. All the nonlinear models can detect the signal from the noise with similar PSD, while linear models such as the AR model missed such detection.

There was no theory of performance limits. All detection theory in the literature was for solving detection of known signals. What we did is to detect chaotic signals with known dynamics but unknown waveforms of the signal. The known dynamics in our experiments were: the driven Duffing System and a 15 dimensional AR model driven by white noise. We generated the time series, fit them with different models, and then used the LRT as a detector in computer simulations. We think there is a relationship between the source and ROC performance which needs to be explained.

There are still two points left for future investigation:

- The choice of modeling technology (HMMs vs. polynomials, splines, etc.) is ad hoc. 
- There is still no demonstration that real signals from important applications have exploitable chaotic characteristics. We would like to find such characteristics in an ongoing application, and then be able to improve system performance. 


\section{REFERENCES}

[1] A. B. Poritz. Hidden markov models: A guided tour. Proc. IEEE Intl. Conf. on Acoust. Speech and Signal Proc, pages 7-13, 1988.

[2] A. M. Fraser and Alexis Dimitriadis. Predicting the Future and Understanding the Past: a Comparision of Approaches, chapter Hidden Markov Models with Mixed States. Addison Wesley, 1993.

[3] H. L. Van Trees. Detection, Estimation, and Modulation Theory. J. Wiley, New York, 1968.

[4] J. G. Lucas D. J. Defatta and W. S. Hodgkiss. Digital Signal Processing: A System Design Approach. J. Wiley, 1988.

[5] M. D. Srinath and P. K. Rajasekaran. An Introduction to Statistical Signal Processing With Applications. J. Wiley, 1979.

[6] S. M. Kay. Modern spectral estimation: theory $\&$ application. Prentice Hall, Englewood Cliffs, New Jersey 07632, NY, 1988.

[7] A. V. Oppenheim and R. W. Schafer. Discrete-Time Signal Processing. Prentice-Hall, Englewood Cliffs, NJ, 1989.

[8] R. Shaw. The dripping faucet as a model chaotic system. University of California, Santa Cruz, CA.

[9] A. Papoulis. Probability random variables, and stochastic process. McGraw-Hill Book Company, Polytechnic Institute of New York, second edition, 1984.

[10] A. M. Fraser and Q. Cai. Detecting chaotic signals with nonlinear models. In Proc. IEEE SSAP Workshop, Victoria BC, 1992.

[11] A. M. Fraser. Reconstructing attractors from scalar time series: A comparison of singular system and redundancy criteria. In Physica $D$, volume $34 \mathrm{D}$, pages 391-404, 1989. 
[12] J. Yorke T. Sauer and M. Casdagli. Embedology. In J. Stat. Phys., volume 65, pages 579-616, 1991.

[13] J. Farmer M. Casdagli, S. Eubank and J. Gibson. State space reconstruction in the presence of noise. In Physica D, volume 51D, pages 52-98, 1991.

[14] L. E. Baum and J. A. Eagon. An inequality with applications to statistical estimation for probabilistic function of markov process and to a model for ecology. Bulletin of the American Mathematical Soviety, pages 73(3):360-363, May 1967.

[15] G. Soules L. E. Baum, T. Petrie and N. Weiss. A maximization technique occurring in the statistical analysis of probabilistic functions of markov chains. Ann. Math. Stat., 41:164-171, 1970.

[16] S. A. Teukolsky W. H. Press, B. P. Flannery and W. T. Veterling. Numerical Recipes in C. Cambridge University Press, Cambridge, 1988.

[17] A. Gersho and R. M. Gray. Vector Quantization and Signal Compression. Norwell MA: Kluwer, 1992.

[18] J. D. Farmer N. H. Packard, J. P. Crutchfiled and R. S. Shaw. Geometry from a time series. In Phys. Rev. Lett., volume 45(9), pages 712-716, September 1980. 